\title{
NONLOCAL QUANTITATIVE ISOPERIMETRIC INEQUALITIES
}

\author{
AGNESE DI CASTRO, MATTEO NOVAGA, BERARDO RUFFINI, AND ENRICO VALDINOCI
}

\begin{abstract}
We show a quantitative-type isoperimetric inequality for fractional perimeters where the deficit of the $t$-perimeter, up to moltiplicative constants, controls from above that of the $s$-perimeter, with $s$ smaller than $t$. To do this we consider a problem of independent interest: we characterize the volume-constrained minimizers of a nonlocal free energy given by the difference of the $t$-perimeter and the $s$-perimeter. In particular, we show that balls are the unique minimizers if the volume is sufficiently small, depending on $t-s$, while the existence vs. nonexistence of minimizers for large volumes remains open. We also consider the corresponding isoperimetric problem and prove existence and regularity of minimizers for all $s, t$. When $s=0$ this problem reduces to the fractional isoperimetric problem, for which it is well known that balls are the only minimizers.
\end{abstract}

\section{Contents}

1. Introduction 1

2. General properties of fractional perimeters 5

3. Preliminary estimates on the energy functional 10

\begin{tabular}{|ll}
\hline $4 . \quad$ Existence of minimizers & 19
\end{tabular}

5. Regularity of minimizers 23

6. Rigidity of minimizers for small volumes and proof of the main theorem 28

7. A fractional isoperimetric problem 31

References

\section{INTRODUCTION}

In this paper we deal with two nonlocal isoperimetric problems, which are closely related one with the other. To introduce them, we recall the definition and some properties of the fractional perimeter. Given a number $\alpha \in(0,1)$, for a measurable set $E \subset \mathbb{R}^{N}$, the fractional perimeter $P_{\alpha}(E)$ is defined as the (squared) $H^{\alpha / 2}$-seminorm of the characteristic function of $E$, that is,

$$
P_{\alpha}(E):=\frac{1}{2}\left[\chi_{E}\right]_{H^{\alpha / 2}}^{2}=\frac{1}{2} \int_{\mathbb{R}^{N}} \int_{\mathbb{R}^{N}} \frac{\left|\chi_{E}(x)-\chi_{E}(y)\right|^{2}}{|x-y|^{N+\alpha}} d x d y=\int_{E} \int_{E^{c}} \frac{d x d y}{|x-y|^{N+\alpha}} .
$$

The notion of fractional perimeter has been introduced in [36, 9] and it has been extensively studied in several recent papers (see for instance [24, 33, 34, 11, 18, 15] and references therein). In particular, according [10, Theorem 1] (see also [7, 14, 3]), we have that the fractional perimeter $P_{\alpha}$, if suitably renormalized, approaches the classical perimeter $P$ as $\alpha \nearrow 1$. More precisely, if $\partial E$ is of class $C^{1, \gamma}$ for some $\gamma>0$, we have

$$
\lim _{\alpha \rightarrow 1^{-}}(1-\alpha) P_{\alpha}(E)=N \omega_{N} P(E),
$$


where $\omega_{N}$ denotes the volume of the $N$-dimensional ball of radius 1 . On the other hand, the fractional perimeter $P_{\alpha}$ approaches the Lebesgue measure $|\cdot|$ as $\alpha \searrow 0$, that is,

$$
\lim _{\alpha \rightarrow 0^{+}} \alpha P_{\alpha}(E)=N \omega_{N}|E|,
$$

if $P_{\bar{\alpha}}(E)<+\infty$ for some $\bar{\alpha}>0$ (see [31] and [17, Corollary 2.6]).

To introduce the first problem we consider we define, for $t \in(0,1)$, the isoperimetric deficit of the $t$-perimeter by

$$
\delta P_{t}(E):=\frac{P_{t}(E)-P_{t}\left(B_{E}\right)}{P_{t}\left(B_{E}\right)}
$$

where $B_{E}$ is a ball of measure $|E|$. The fractional isoperimetric inequality, stating that among sets of fixed measure the ball minimizes the fractional perimeter, reads in term of the isoperimetric deficit as

$$
\delta P_{t}(E) \geqslant 0
$$

Notice that for any $t \in(0,1)$ the isoperimetric deficit is a 0 homogeneous quantity. Moreover thanks to (1.1) and 1.2), for $t \rightarrow 1$ and $s \rightarrow 0$ it converges to the classical deficit (see for instance [21])

$$
\delta P(E)=\frac{P(E)-P\left(B_{E}\right)}{P\left(B_{E}\right)},
$$

and to 0 respectively. In the last years there has been a renewed interest into the study of quantitative stability isoperimetric inequalities, which is a stronger versions of the isoperimetric inequality of the form

$$
\delta P(E) \geqslant \phi(E),
$$

where $\phi(E)$ is a non-negative quantity which measures the distance between the set $E$ and the set of the balls contained in $\mathbb{R}^{N}$. A cornerstone example has been given in the paper [23] where the authors show an inequality of the form

$$
\delta P(E) \geqslant C_{N} \alpha(E):=C_{N} \min _{x \in \mathbb{R}^{N}} \frac{\left|E \Delta\left(B_{E}+x\right)\right|^{2}}{|E|},
$$

proving that the exponent 2 is asimptotically optimal, as $E$ approaches $B_{E}$. Here $C_{N}$ is a dimensional constant while $|E \Delta F|$ indicates the Lebesgue measure of the symmetric difference between $E$ and $F$. The quantity $\alpha(E)$ is usually referred to as Fraenkel asymmetry. Recently the (sharp) fractional counterpart of (1.4) has been shown in [18. Namely it is proved that there exists a constant $C_{N, t}$ such that for any $E \subset \mathbb{R}^{N}$ it holds

$$
\delta P_{t}(E) \geqslant C_{N, t} \alpha(E)^{2} .
$$

Here, again the exponent 2 is optimal.

The first main result of this paper is the following.

Theorem 1.1. Let $0<s<t<1$. Then there exists a constant $C(N, s, t)$ such that for any $E \subset \mathbb{R}^{N}$ the following inequality holds true

$$
\delta P_{t}(E) \geqslant C(N, s, t) \delta P_{s}(E) .
$$

Moreover the constant $C(N, s, t)$ is bounded as $s \rightarrow 0$ and $t \rightarrow 1$. 
Some comments about the proof of Theorem 1.1 are in order. First we notice that in view of (1.5), inequality (1.6) might be seen as a stronger version of the quantitative isoperimetric inequality. To get 1.6 we investigate another variational problem:

$$
\min _{|E|=m} \mathcal{F}_{s, t}(E) \quad m \in(0,+\infty),
$$

where

$$
\mathcal{F}_{s, t}(E):= \begin{cases}(1-t) P_{t}(E)-s P_{s}(E) & \text { if } 0<s<t<1 \\ N \omega_{N} P(E)-s P_{s}(E) & \text { if } 0<s<t=1 \\ (1-t) P_{t}(E)-N \omega_{N}|E| & \text { if } 0=s<t<1 \\ N \omega_{N} P(E)-N \omega_{N}|E| & \text { if } s=0 \text { and } t=1 .\end{cases}
$$

Notice that thanks to $(1.2)$ and $(1.1)$, for all $s, t \in(0,1)$ we have

$$
\mathcal{F}_{s, t}(E) \underset{t \rightarrow 1}{\rightarrow} \mathcal{F}_{s, 1}(E) \underset{s \rightarrow 0}{\rightarrow} \mathcal{F}_{0,1}(E) \quad \text { and } \quad \mathcal{F}_{s, t}(E) \underset{s \rightarrow 0}{\rightarrow} \mathcal{F}_{0, t}(E) \underset{t \rightarrow 1}{\rightarrow} \mathcal{F}_{0,1}(E),
$$

that is, $\mathcal{F}_{s, t}$ depends continuously on $s, t \in[0,1]$, with $s<t$.

Problem (1.7) is, in our opinion, of independent interest as it is reminiscent of recent results about isoperimetric problems with nonlocal competing term arising in mathematical physics, where the functionals take the form

$$
\mathcal{F}=P+\mathcal{N} \mathcal{L}
$$

being $P$ the perimeter and $\mathcal{N} \mathcal{L}$ the nonlocal term, see for instance $[28,29,13,26,22,18,15,27$. We mention in particular the works by Knüpfer and Muratov [28, 29] where the authors consider the case where the nonlocal term is given by a Coulombic potential. In our framework, the energy in 1.9 presents a competing effect between the term $P_{t}$ which has the tendency to "aggregate" the sets into balls, and $P_{s}$, which acts in the opposite way. We will see that, at small scales, the aggregating effect is predominant, but this does not occur at large scales. More precisely, as a first result we show that minimizers exist and are regular at least for small volumes.

Theorem 1.2. For any $0 \leqslant s<t \leqslant 1$, there exists $\bar{m}_{0}=\bar{m}_{0}(N, t-s)>0$ such that for all $m \in\left(0, \bar{m}_{0}\right)$, problem (1.7) has a minimizer $F \subset \mathbb{R}^{N}$. Moreover $F$ is bounded with boundary of class $C^{1, \beta}$, for some $\beta=\beta(N, t-s) \in(0,1)$, outside a closed singular set of Hausdorff dimension at most $N-2$ (respectively $N-8$ if $t=1)$.

Exploiting the fractional isoperimetric inequality in a quantitative form proved in [18, we then show that the the minimizer found in Theorem 1.2 is necessarily a ball, if the volume $m$ is sufficiently small.

Theorem 1.3. For any $0 \leqslant s<t \leqslant 1$ and $\bar{m}_{0}$ as in Theorem 1.2, there exists $\bar{m}_{1}=\bar{m}_{1}(N, t-$ $s) \in\left(0, \bar{m}_{0}\right]$ such that for all $m \in\left(0, \bar{m}_{1}\right)$, the only minimizer of problem (1.7) is given by the ball of measure $m$.

Once Theorem 1.3 is settled, the proof of Theorem 1.1 easily follows. We stress that our estimates, similarly to those in [18], depend only on a lower bound on the difference $t-s$, and pass to the limit as $s \rightarrow 0$ and $t \rightarrow 1$ (as a matter of fact, the normalizing constants appearing in (1.8) has exactly the purpose of making our estimates stable as $s \rightarrow 0$ and $t \rightarrow 1$ ). Moreover, 
as far as we know, our results are new even in the case $t=1$. We also point out that we do not know if a minimizer exists for any volume $m$. However, we show that a minimizer cannot be a ball if $m$ is large enough (see Theorem 6.3), so the minimization problem can be in general quite rich.

The second problem we consider is the following generalized isoperimetric problem:

$$
\min _{E \subset \mathbb{R}^{N}} \widetilde{\mathcal{F}_{s, t}}(E) \quad 0 \leqslant s<t \leqslant 1
$$

where

$$
\widetilde{\mathcal{F}_{s, t}}(E):= \begin{cases}\frac{\left((1-t) P_{t}(E)\right)^{N-s}}{\left(s P_{s}(E)\right)^{N-t}} & \text { if } 0<s<t<1 \\ \frac{\left(N \omega_{N} P(E)\right)^{N-s}}{\left(s P_{s}(E)\right)^{N-1}} & \text { if } 0<s<t=1 \\ \frac{(1-t) P_{t}(E)^{N}}{\left(N \omega_{N}|E|\right)^{N-t}} & \text { if } 0=s<t<1 \\ N \omega_{N} \frac{P(E)^{N}}{|E|^{N-1}} & \text { if } s=0 \text { and } t=1 .\end{cases}
$$

Again, thanks to $(1.2)$ and 11.1 we see that

$$
\widetilde{\mathcal{F}_{s, t}}(E) \underset{t \rightarrow 1}{\rightarrow} \widetilde{\mathcal{F}_{s, 1}}(E) \underset{s \rightarrow 0}{\rightarrow} \widetilde{\mathcal{F}_{0,1}}(E) \quad \text { and } \quad \widetilde{\mathcal{F}_{s, t}}(E) \underset{s \rightarrow 0}{\rightarrow} \widetilde{\mathcal{F}_{0, t}}(E) \underset{t \rightarrow 1}{\rightarrow} \widetilde{\mathcal{F}_{0,1}}(E) \text {. }
$$

Since, for $s=0$ and $t=1$, problem 1.10 reduces to the classical isoperimetric one, while for $t<1$ it reduces to the fractional isoperimetric one, we can think to it as a generalized isoperimetric problem for fractional perimeters. Moreover in the cases $s=0<t \leqslant 1$ it is well known that the ball is the unique minimizer of $\widetilde{\mathcal{F}_{s, t}}$. Nevertheless we don't know if the ball minimizes $\widetilde{\mathcal{F}_{s, t}}$ for any $0<s<t \leqslant 1$. Our main result about problem 1.10 is the following.

Theorem 1.4. For any $0 \leqslant s<t \leqslant 1$, there exists a nontrivial minimizer $E_{s, t}$ of problem 1.10$)$. Moreover $E_{s, t}$ is bounded and has boundary of class $C^{1, \beta}$, for some $\beta=\beta(N, t-s) \in(0,1)$, outside a closed singular set of Hausdorff dimension at most $N-2$ (respectively $N-8$ if $t=1)$.

Remark 1.5. An observation which may support the conjecture that the ball is a minimizer of $\widetilde{\mathcal{F}_{s, t}}$ is the following link between inequality 1.6 and problem 1.10. By the concavity of the map $x \rightarrow(1+x)^{(N-t) /(N-s)}$, if the quantity $\delta P_{s}(E)$ is not too large, then

$$
\left(1+\delta P_{s}(E)\right)^{(N-t) /(N-s)} \leqslant 1+\frac{N-t}{N-s} \delta P_{s}(E) .
$$

Moreover a straightforward computation shows that the inequality

$$
\widetilde{\mathcal{F}_{s, t}}(E) \geqslant \widetilde{\mathcal{F}_{s, t}}(B)
$$

is equivalent to the following one

$$
1+\delta P_{t}(E) \geqslant\left(1+\delta P_{s}(E)\right)^{\frac{N-t}{N-s}} .
$$

Thus, the mixed isoperimetric problem (1.10) has as solution the ball if the mixed quantitative fractional isoperimetric inequality (1.6) holds true with a constant $C(N, s, t)$ greater or equal than $(N-t) /(N-s)$. 
We remark that the problems considered in this paper and some techniques exploited to get the proof of Theorem 1.1 are related to the very recent paper [18, since both here and there some nonlocal functionals built by the combination of aggregating and disaggregating terms are studied via variational methods and geometric measure theory techniques. Nevertheless there are several technical and conceptual differences between our case and the one of [18]. For instance, the disaggregating term in [18] comes from a Riesz potential (i.e. it has a locally integrable kernel), and the minimizers of such functional are $\Lambda$-minimizers for the aggregating term (i.e. their energy surplus reduces to a volume perturbation). On the contrary, our disaggregating terms have somehow the same type of nonlocal structure as the aggregating ones (for instance they do not come from a locally integrable kernel), and our minimizers are only $\omega$-minimizers of the fractional perimeter (hence their rigidity and regularity properties are less standard). Also, our techniques are different than the ones in [18; for instance, we highly rely on a relative isoperimetric inequality (see Lemma 2.5) and on conceptually different regularity results (see e.g. the second inequality in (6.2).

About the proof of Theorem 1.4, if the regularity results presented are basically a straightforward application of already developed tools in Geometric Measure Theory, the existence issue, mainly because of the competition between the numerator and the denominator in the definition of $\widetilde{\mathcal{F}_{s, t}}$, is less straightforward. Indeed, to get the existence part of the proof of Theorem 1.4 . it is necessary a quite more original and non-trivial approach. For the reader convenience we added a formal description of the strategy of the proof at the beginning of Section 7 .

The paper is organized as follows: in Section 2 we recall and prove some general properties of the fractional perimeters and, more generally, of the fractional Sobolev seminorms. In Sections 3 6 we deal with problem (1.7). Section 3 contains the main tools exploited later to prove Theorems 1.2 and 1.3 . The cornerstone of the section is an optimality criterion (see Proposition 3.9) which entails density estimates for minimizers (see Proposition 3.11) and the fact that minimizers must be close to a ball, if the volume is small enough (see Lemma 3.13). An elementary, but important result is then provided by Proposition 3.12, stating that any minimum must be necessary bounded and, if $t=1$ (that is, $\mathcal{F}_{s, 1}=N \omega_{N} P-s P_{s}$ ), also essentially connected. Section 4 contains Theorem 4.2, which solves the existence part of Theorem 1.2, while in Section 5 we prove that any minimizer has smooth boundary, out of a closed singular set. Then, in Section 6 we show that, if the volume $m$ is below a certain threshold $\bar{m}_{1}>0$, the ball is the unique minimizer for problem (1.7) and we give the proof of Theorem 1.1. Eventually, in Section 7, we deal with problem (1.10). The main result here is given by Theorem 1.4, where we show the existence and regularity of minimizers.

\section{General Properties of Fractional Perimeters}

Before starting to prove some properties of fractional perimeters it is convenient to fix some notation which will be used throughout the rest of the paper. Firstly, notice that we will denote by $c_{N}$ a general positive constant depending only on the dimension $N$ and by $c_{0}$ a positive constant depending on $N$ and $\delta_{0}$ a fixed quantity such that $0<\delta_{0} \leqslant t-s$, which will not necessarily be the same at different occurrences and which can also change from line to line; special constants will be denoted by $c_{1}, c_{2}, \ldots$ Relevant dependences on parameters will be emphasized by using parentheses.

As customary, we denote by $B\left(x_{0}, R\right):=\left\{x \in \mathbb{R}^{N}:\left|x-x_{0}\right|<R\right\}$ the open ball centered in $x_{0} \in \mathbb{R}^{N}$ with radius $R>0$. We shall use the shorter notation $B=B(0,1)$, with $|B(0,1)|=\omega_{N}$. 
Moreover, when not important and clear from the context, we shall denote by $B_{m}$ the ball of volume $m$, that is of radius $R=(m /|B(0,1)|)^{1 / N}$.

Finally, as usual, given two sets $E$ and $F$ of $\mathbb{R}^{N}$, we denote the symmetric difference between $E$ and $F$ as $E \Delta F=(E \backslash F) \cup(F \backslash E)$.

We begin by a simple result.

Lemma 2.1. Let $E=E_{1} \cup E_{2}$ a subset of $\mathbb{R}^{N}$ with $\left|E_{1} \cap E_{2}\right|=0$. Then

$$
P_{\alpha}(E)=P_{\alpha}\left(E_{1}\right)+P_{\alpha}\left(E_{2}\right)-2 \int_{E_{1}} \int_{E_{2}} \frac{d x d y}{|x-y|^{N+\alpha}}
$$

In particular

$$
P_{\alpha}(E) \leqslant P_{\alpha}\left(E_{1}\right)+P_{\alpha}\left(E_{2}\right)
$$

Proof. Let us denote by $\chi_{E}$ the characteristic function of the set $E$. We have

$$
\begin{aligned}
P_{\alpha}(E) & =\frac{1}{2} \int_{\mathbb{R}^{N}} \int_{\mathbb{R}^{N}} \frac{\left(\chi_{E}(x)-\chi_{E}(y)\right)^{2}}{|x-y|^{N+\alpha}} d x d y \\
& =\frac{1}{2} \int_{\mathbb{R}^{N}} \int_{\mathbb{R}^{N}} \frac{\left(\chi_{E_{1}}(x)+\chi_{E_{2}}(x)-\chi_{E_{1}}(y)-\chi_{E_{2}}(y)\right)^{2}}{|x-y|^{N+\alpha}} d x d y \\
& =\frac{1}{2} \int_{\mathbb{R}^{N}} \int_{\mathbb{R}^{N}} \frac{\left(\chi_{E_{1}}(x)-\chi_{E_{1}}(y)\right)^{2}+\left(\chi_{E_{2}}(x)-\chi_{E_{2}}(y)\right)^{2}}{|x-y|^{N+\alpha}} \\
& +\int_{\mathbb{R}^{N}} \int_{\mathbb{R}^{N}} \frac{\left(\chi_{E_{1}}(x)-\chi_{E_{1}}(y)\right)\left(\chi_{E_{2}}(x)-\chi_{E_{2}}(y)\right)}{|x-y|^{N+\alpha}} d x d y \\
& =P_{\alpha}\left(E_{1}\right)+P_{\alpha}\left(E_{2}\right)-2 \int_{E_{1}} \int_{E_{2}} \frac{d x d y}{|x-y|^{N+\alpha}} .
\end{aligned}
$$

For further use, we also prove the following interpolation estimate (by reasoning as in [8, Proposition 4.2 and Corollary 4.4]):

Lemma 2.2. For any $E \subset \mathbb{R}^{N}$ and $0<s<t<1$ there holds

$$
P_{s}(E) \leqslant c_{N} \frac{1}{s}\left(1-\frac{s}{t}\right)^{-1}|E|^{1-\frac{s}{t}}(1-t)^{\frac{s}{t}} P_{t}(E)^{\frac{s}{t}} .
$$

Proof. We reason as in [8, Prop. 4.2]. Letting $u=\chi_{E}$, we can write

$$
\begin{aligned}
P_{s}(E)= & \frac{1}{2} \int_{\mathbb{R}^{N}} \int_{\mathbb{R}^{N}} \frac{|u(x+h)-u(x)|}{|h|^{N+s}} d x d h \\
= & \frac{1}{2} \int_{|h|<1} \int_{\mathbb{R}^{N}} \frac{|u(x+h)-u(x)|}{|h|^{N+s}} d x d h \\
& +\frac{1}{2} \int_{|h| \geqslant 1} \int_{\mathbb{R}^{N}} \frac{|u(x+h)-u(x)|}{|h|^{N+s}} d x d h=: I_{1}+I_{2} .
\end{aligned}
$$

We recall that, by [8, Lemma A.1] (see also [31]), there exists a constant $c_{N}$ such that

$$
\int_{\mathbb{R}^{N}} \frac{|u(x+h)-u(x)|}{|h|^{t}} d x \leqslant c_{N}(1-t) P_{t}(E),
$$


for all $|h|>0$. We then estimate

$$
\begin{aligned}
I_{1} & =\int_{|h|<1} \int_{\mathbb{R}^{N}} \frac{|u(x+h)-u(x)|}{|h|^{N+s}} d x d h \\
& \leqslant c_{N}(1-t) P_{t}(E) \int_{|h|<1} \frac{1}{|h|^{N-(t-s)}} d h \\
& =c_{N} \frac{1-t}{t-s} P_{t}(E),
\end{aligned}
$$

and

$$
\begin{aligned}
I_{2} & =\int_{|h| \geqslant 1} \int_{\mathbb{R}^{N}} \frac{|u(x+h)-u(x)|}{|h|^{N+s}} d x d h \\
& \leqslant 2|E| \int_{|h| \geqslant 1} \frac{1}{|h|^{N+s}} d h \\
& =\frac{2 N \omega_{N}}{s}|E| .
\end{aligned}
$$

Putting together 2.5) and 2.6 we then get, up to rename $c_{N}$,

$$
P_{s}(E) \leqslant c_{N} \frac{1-t}{t-s} P_{t}(E)+\frac{N \omega_{N}}{s}|E| .
$$

If we evaluate (2.7) on the set $\lambda E$, with $\lambda>0$, we obtain

$$
\lambda^{N-s} P_{s}(E) \leqslant c_{N} \frac{1-t}{t-s} \lambda^{N-t} P_{t}(E)+\lambda^{N} \frac{N \omega_{N}}{s}|E|,
$$

that is,

$$
\lambda^{t-s} P_{s}(E)-\lambda \frac{N \omega_{N}|E|}{s} \leqslant \frac{c_{N}(1-t)}{t-s} P_{t}(E) .
$$

The expression at the left-hand side of 2.8 reaches its maximum at

$$
\lambda=\left(\frac{s(t-s) P_{s}(E)}{2 N \omega_{N} t|E|}\right)^{\frac{1}{s}} .
$$

Substituting this value of $\lambda$ into 2.8 we get 2.3.

Remark 2.3. If we let $t \rightarrow 1^{-}$in $(2.3)$, we recover the estimate in [8, Cor. 4.4]:

$$
P_{s}(E) \leqslant \frac{c_{N}}{s(1-s)}|E|^{1-s} P(E)^{s} .
$$

Indeed the proof of Lemma 2.2 extends to the case $t=1$, by substituting $(1-t) P_{t}(E)$ with $P(E)$ in the right hand side of $(2.4)$.

We show now a version of the local fractional isoperimetric inequality. For this, we recall that the fractional perimeter of a set $E$ in a bounded set $\Omega$ is defined by

$$
P_{\alpha}(E, \Omega):=\int_{E \cap \Omega} \int_{\mathbb{R}^{N} \backslash E} \frac{d x d y}{|x-y|^{N+\alpha}}+\int_{\Omega \backslash E} \int_{E \backslash \Omega} \frac{d x d y}{|x-y|^{N+\alpha}} .
$$

With this setting, we have a variant of Lemma 2.1 as follows:

Lemma 2.4. Let $\Omega_{1}$ and $\Omega_{2}$ be disjoint bounded sets. Then

$$
P_{\alpha}\left(E, \Omega_{1}\right)+P_{\alpha}\left(E, \Omega_{2}\right) \leqslant P_{\alpha}\left(E, \Omega_{1} \cup \Omega_{2}\right)+2 \int_{\Omega_{1}} \int_{\Omega_{2}} \frac{d x d y}{|x-y|^{N+\alpha}} .
$$


Proof. We use 2.10 (omitting the integrands for simplicity) to compute

$$
\begin{aligned}
& P_{\alpha}\left(E, \Omega_{1} \cup \Omega_{2}\right)-P_{\alpha}\left(E, \Omega_{1}\right)-P_{\alpha}\left(E, \Omega_{2}\right) \\
= & \int_{E \cap\left(\Omega_{1} \cup \Omega_{2}\right)} \int_{\mathbb{R}^{N} \backslash E}+\int_{\left(\Omega_{1} \cup \Omega_{2}\right) \backslash E} \int_{E \backslash\left(\Omega_{1} \cup \Omega_{2}\right)}-\int_{E \cap \Omega_{2}} \int_{\mathbb{R}^{N} \backslash E}-\int_{\Omega_{2} \backslash E} \int_{E \backslash \Omega_{1}}-\int_{\mathbb{R}_{1} \backslash E}-\int_{E \backslash \Omega_{1}}+\int_{\Omega_{2} \backslash E} \int_{E \backslash\left(\Omega_{1} \cup \Omega_{2}\right)} \\
= & \int_{E \cap \Omega_{1}} \int_{\mathbb{R}^{N} \backslash E}+\int_{E \cap \Omega_{2}} \int_{\mathbb{R}^{N} \backslash E}+\int_{\Omega_{1} \backslash E} \int_{E \backslash\left(\Omega_{1} \cup \Omega_{2}\right)}+\int_{\Omega_{1} \backslash E} \int_{E \backslash \Omega_{1}}-\int_{E \cap \Omega_{2}} \int_{\mathbb{R}^{N} \backslash E}-\int_{\Omega_{2} \backslash E} \int_{E \backslash \Omega_{1}}-\int_{\Omega_{1} \backslash E} \int_{E \backslash \Omega_{1}}-\int_{\Omega_{2} \backslash E} \int_{E \backslash \Omega_{1}} \\
= & \int_{\Omega_{1} \backslash E} \int_{E \backslash\left(\Omega_{1} \cup \Omega_{2}\right)}+\int_{\Omega_{2} \backslash E} \int_{E \backslash\left(\Omega_{1} \cup \Omega_{2}\right)}-\int_{\mathbb{R}^{N} \backslash E}-\int_{\Omega_{2} \backslash E} \int_{\left(E \backslash \Omega_{2}\right) \cap \Omega_{1}} .
\end{aligned}
$$

This implies 2.11).

Then, we have the following local fractional isoperimetric inequality:

Lemma 2.5. Let $\Omega$ be a open bounded set with Lipschitz boundary and let $E \subseteq \mathbb{R}^{N}$ such that $|E \cap \Omega|<|\Omega| / 2$. Then there exists a constant $C=C(|\Omega|, N, \alpha)$ such that

$$
P_{\alpha}(E, \Omega) \geqslant C|E \cap \Omega|^{\frac{N-\alpha}{N}} \text {. }
$$

Proof. The case $t=1$ is classical. For its proof we refer to [30, Section II.1.6]. We begin by recalling the Poincaré-type inequality for fractional Sobolev spaces (see for instance [7, Equations (2) and (3)]): for any $p \geqslant 1$ and $\alpha \in(0,1)$, given a function $f \in L^{p}(\Omega)$ we have that

$$
\int_{\Omega} \int_{\Omega} \frac{|f(x)-f(y)|^{p}}{|x-y|^{N+\alpha p}} \geqslant C(N, \alpha, p,|\Omega|)\left\|f-f_{\Omega}\right\|_{L^{q}(\Omega)}^{p}
$$

where

$$
f_{\Omega}=\frac{1}{|\Omega|} \int_{\Omega}|f| d x
$$

and

$$
\frac{1}{q}=\frac{1}{p}-\frac{\alpha}{N}
$$


By applying (2.13) with $p=1, \alpha \in(0,1)$ and $f=\chi_{E}$, and by the very definition of $P_{\alpha}(E)$ we get that

$$
\begin{aligned}
2 P_{\alpha}(E, \Omega) & \geqslant \int_{\Omega} \int_{\Omega} \frac{\left|\chi_{E}(x)-\chi_{E}(y)\right|}{|x-y|^{N+\alpha}} \\
& \geqslant C(N, \alpha,|\Omega|)\left(\int_{\Omega}\left|\chi_{E}(x)-\frac{|E \cap \Omega|}{|\Omega|}\right|^{q} d x\right)^{1 / q} \\
& =C(N, \alpha,|\Omega|)\left[|E \cap \Omega|\left(1-\frac{|E \cap \Omega|}{|\Omega|}\right)^{q}+|\Omega \backslash E|\left(\frac{|E \cap \Omega|}{|\Omega|}\right)^{q}\right]^{1 / q} \\
& \geqslant C(N, \alpha,|\Omega|)|E \cap \Omega|^{1 / q}\left(1-\frac{|E \cap \Omega|}{|\Omega|}\right) \\
& \geqslant \frac{C(N, \alpha,|\Omega|)}{2}|E \cap \Omega|^{1 / q} .
\end{aligned}
$$

Since, by (2.14), $q=N /(N-\alpha)$, the proof is concluded.

Beside the local fractional isoperimetric inequality (2.12), we recall from [19] the standard (fractional) one: if $0<t_{0} \leqslant \alpha \leqslant 1$ then it holds (if $|E|<+\infty$ )

$$
(1-\alpha) P_{\alpha}(E) \geqslant c\left(N, t_{0}\right)|E|^{\frac{N-\alpha}{N}}, \quad \text { where } c\left(N, t_{0}\right)=\frac{c_{N}}{t_{0}},
$$

We now recall some basic facts on hypersingular Riesz operators on the sphere, following [18, pp. 4-5] (see also [32, pp. 159-160]). We denote by $\mathcal{S}_{k}$ the space of spherical harmonics of degree $k$, and by $d(k)$ the dimension of $\mathcal{S}_{k}$. For $\alpha \in(0,1)$ we also let $\mathcal{J}_{\alpha}$ be the operator defined as

$$
\mathcal{J}_{\alpha} u(x)=2 \text { p.v. } \int_{\partial B} \frac{u(x)-u(y)}{|x-y|^{N+\alpha}} d \mathcal{H}^{N-1}(y) \quad \text { for } u \in C^{2}(\partial B),
$$

(with the symbol p.v. we mean that the integral is considered in the Cauchy principal value sense) and we let $\lambda_{k}^{\alpha}$ be the $k^{\text {th }}$ eigenvalue of $\mathcal{J}_{\alpha}$, that is,

$$
\mathcal{J}_{\alpha} Y=\lambda_{k}^{\alpha} Y \quad \text { for any } Y \in \mathcal{S}_{k} .
$$

We then have $\lambda_{k}^{\alpha} \rightarrow+\infty$ as $k \rightarrow+\infty$, and

$$
\lambda_{0}^{\alpha}=0 \quad \lambda_{k+1}^{\alpha}>\lambda_{k}^{\alpha} \quad \forall k \in \mathbb{N} \cup\{0\} .
$$

If we let $\left\{Y_{k}^{i}\right\}_{i=1}^{d(k)}$ be an orthonormal basis of $\mathcal{S}_{k}$ in $L^{2}(\partial B)$, and denote by

$$
a_{k}^{i}(u):=\int_{\partial B} u Y_{k}^{i} d \mathcal{H}^{N-1},
$$

the Fourier coefficients of $u \in L^{2}(\partial B)$ corresponding to $Y_{k}^{i}$, we have

$$
\begin{aligned}
{[u]_{H^{\frac{1+\alpha}{2}}(\partial B)}^{2} } & :=\int_{\partial B} \int_{\partial B} \frac{|u(x)-u(y)|^{2}}{|x-y|^{N+\alpha}} d \mathcal{H}^{N-1}(x) d \mathcal{H}^{N-1}(y) \\
& =\int_{\partial B} u \mathcal{J}_{\alpha} u d \mathcal{H}^{N-1} \\
& =\sum_{k=0}^{\infty} \sum_{i=0}^{d(k)} \lambda_{k}^{\alpha} a_{k}^{i}(u)^{2} .
\end{aligned}
$$


Proposition 2.6. ([18, Proposition 2.3]) We have

$$
\lambda_{k}^{\alpha} \geqslant \lambda_{1}^{\alpha}=\alpha(N-\alpha) \frac{P_{\alpha}(B)}{P(B)} \geqslant \frac{1}{c_{N}(1-\alpha)} .
$$

Proposition 2.7. Let $u \in H^{\frac{1+t}{2}}(\partial B)$ and $0 \leqslant s \leqslant t<1$ then the following estimate holds

$$
(1-s)[u]_{H^{\frac{1+s}{2}}(\partial B)}^{2} \leqslant c_{N}(1-t)[u]_{H^{\frac{1+t}{2}}}^{2}(\partial B) .
$$

Proof. By (2.16) and using the estimate for $\lambda_{k}$ established in Proposition 2.6 we get

$$
\begin{aligned}
(1-s)[u]_{H^{\frac{1+s}{2}}(\partial B)}^{2} & =(1-s) \sum_{k=0}^{\infty} \sum_{i=0}^{d(k)} \lambda_{k}^{s} a_{k}^{i}(u)^{2}=(1-s) \sum_{k=0}^{\infty} \sum_{i=0}^{d(k)} \lambda_{k}^{s-t} \lambda_{k}^{t} a_{k}^{i}(u)^{2} \\
& \leqslant(1-s) \lambda_{1}^{s-t} \sum_{k=0}^{\infty} \sum_{i=0}^{d(k)} \lambda_{k}^{t} a_{k}^{i}(u)^{2} \\
& \leqslant(1-s) \lambda_{1}^{s} c_{N}(1-t) \sum_{k=0}^{\infty} \sum_{i=0}^{d(k)} \lambda_{k}^{t} a_{k}^{i}(u)^{2} \\
& =(1-s) s(N-s) \frac{P_{s}(B)}{P(B)} c_{N}(1-t)[u]_{H^{\frac{1+t}{2}}(\partial B)}^{2} \\
& \leqslant c_{N}(1-t)[u]_{H^{\frac{1+t}{2}}}^{2}(\partial B)
\end{aligned}
$$

Remark 2.8. We note that the result established in the previous proposition remains true also in the case $t=1$. Indeed, since

$$
\lim _{t \rightarrow 1^{-}}(1-t)[u]_{H^{\frac{1+t}{2}}(\partial B)}^{2}=[u]_{H^{1}(\partial B)}^{2}
$$

as established in [6, Cor. 2], we can pass to the limit $t \rightarrow 1^{-}$in (2.17).

\section{Preliminary estimates on the energy functional}

In the following we shall consider parameters $s, t \in(0,1)$ satisfying

$$
t-s \geqslant \delta_{0}>0 \text {. }
$$

All the constants in this work, unless differently specified, will depend only on $N$ and $\delta_{0}$, so that it will be possible to pass to the limits in a straightforward way as $s \rightarrow 0^{+}$or $t \rightarrow 1^{-}$.

Proposition 3.1. There exists $c_{0}=c_{0}\left(N, \delta_{0}\right)$ such that, for any $E \subset \mathbb{R}^{N}$ and $0<s<t<1$ satisfying (3.1), it holds

$$
\mathcal{F}_{s, t}(E) \geqslant \frac{(1-t) P_{t}(E)}{2}-c_{0}|E| \text {. }
$$

Proof. Set $m:=|E|$. We apply Young inequality with exponents $\frac{t}{t-s}$ and $\frac{t}{s}$ to the right hand side of (2.3) getting

$$
\begin{aligned}
c_{N} \frac{1}{s}\left(1-\frac{s}{t}\right)^{-1}|E|^{1-\frac{s}{t}}(1-t)^{\frac{s}{t}} P_{t}(E)^{\frac{s}{t}} & =\left[c_{N} \frac{2^{\frac{s}{t}}}{s}\left(1-\frac{s}{t}\right)^{-1} m^{1-\frac{s}{t}}\right]\left[2^{-1}(1-t) P_{t}(E)\right]^{\frac{s}{t}} \\
& \leqslant\left[c_{N} \frac{2^{\frac{s}{t}}}{s}\left(1-\frac{s}{t}\right)^{-1} m^{1-\frac{s}{t}}\right]^{\frac{t}{t-s}}+\frac{(1-t) P_{t}(E)}{2} .
\end{aligned}
$$


Thus 2.3 gives that

$$
\begin{aligned}
\mathcal{F}_{s, t}(E) & =(1-t) P_{t}(E)-s P_{s}(E) \geqslant(1-t) P_{t}(E)-c_{N}\left(1-\frac{s}{t}\right)^{-1}|E|^{1-\frac{s}{t}}(1-t)^{\frac{s}{t}} P_{t}(E)^{\frac{s}{t}} \\
& \geqslant(1-t) P_{t}(E)-\left[2^{\frac{s}{t}} c_{N}\left(1-\frac{s}{t}\right)^{-1} m^{1-\frac{s}{t}}\right]^{\frac{t}{t-s}}-\frac{(1-t) P_{t}(E)}{2} \\
& =\frac{(1-t) P_{t}(E)}{2}-\left[2^{\frac{s}{t}} c_{N} \frac{t}{t-s}\right]^{\frac{t}{t-s}} m
\end{aligned}
$$

and this concludes the proof.

Corollary 3.2. Let $|E|=m$. Then both $P_{t}(E)$ and $P_{s}(E)$ are bounded above by quantities only depending on $m$ and $\mathcal{F}_{s, t}(E)$. More explicitly

$$
\begin{array}{ll} 
& (1-t) P_{t}(E) \leqslant 2\left(\mathcal{F}_{s, t}(E)+c_{0} m\right) \\
\text { and } \quad & s P_{s}(E) \leqslant c_{0}^{1-\frac{s}{t}} m^{1-\frac{s}{t}}\left(\mathcal{F}_{s, t}(E)+c_{0} m\right)^{\frac{s}{t}},
\end{array}
$$

with $c_{0}$ as in Proposition 3.1.

Proof. We obtain (3.3) easily from Proposition 3.1. Then (3.4) follows from (2.3) and (3.3).

Now we define the isovolumetric function $\phi:(0,+\infty) \rightarrow \mathbb{R}$ as

$$
\phi(m)=\inf _{|E|=m} \mathcal{F}_{s, t}(E) \quad m \in(0,+\infty) .
$$

A general estimate on $\phi(m)$ goes as follows:

Lemma 3.3. We have

$$
-c_{0} m \leqslant \phi(m) \leqslant c_{1} m^{\frac{N-t}{N}}\left(1-\frac{c_{2}}{c_{1}} m^{\frac{t-s}{N}}\right),
$$

with $c_{0}$ as in Proposition 3.1 and

$$
c_{1}:=\frac{(1-t) P_{t}(B)}{|B|^{\frac{N-t}{N}}} \quad \text { and } \quad c_{2}:=\frac{s P_{s}(B)}{|B|^{\frac{N-s}{N}}} .
$$

Proof. Let us begin by proving the estimate from above of $\phi(m)$. For this, we take the unit ball $B$ we set $\rho:=(m /|B|)^{1 / N}$ and we consider the ball $B(0, \rho)$ of radius $\rho$. Notice that $|B(0, \rho)|=$ $\rho^{N}|B|=m$,

$$
P_{t}(B(0, \rho))=\rho^{N-t} P_{t}(B)=\frac{P_{t}(B)}{|B|^{\frac{N-t}{N}}} m^{\frac{N-t}{N}}
$$

and

$$
P_{s}(B(0, \rho))=\rho^{N-s} P_{s}(B)=\frac{P_{s}(B)}{|B|^{\frac{N-s}{N}}} m^{\frac{N-s}{N}} .
$$

By minimality, we get, with $c_{1}$ and $c_{2}$ as in 3.6 ,

$$
\phi(m) \leqslant \mathcal{F}_{s, t}(B(0, \rho))=(1-t) P_{t}(B(0, \rho))-s P_{s}(B(0, \rho))=c_{1} m^{\frac{N-t}{N}}\left(1-\frac{c_{2}}{c_{1}} m^{\frac{t-s}{N}}\right),
$$

that proves 3.5 .

The first inequality in 3.5 follows from Proposition 3.1 . 
Remark 3.4. We recall the fractional isoperimetric inequality, which holds true for any measurable set $E$ such that $|E|<+\infty$ :

$$
|E|^{\frac{N-t}{N}} \leqslant c_{N} t(1-t) P_{t}(E) .
$$

For the optimal constant $c_{N}$ we refer to [20] (see in particular Equations (1.10) and (4.2) there).

Lemma 3.5. There exist $m_{0}=m_{0}\left(N, \delta_{0}\right)$ and $m_{1}=m_{1}\left(N, \delta_{0}\right)$ such that:

$$
\begin{aligned}
& \text { if } m>m_{1} \text {, then } \phi(m)<0 \text {; } \\
& \text { if } m \in\left(0, m_{0}\right) \text {, then } \phi(m) \geqslant \frac{c_{N}}{t} m^{\frac{N-t}{N}}>0 .
\end{aligned}
$$

Moreover

$$
\lim _{m \rightarrow 0^{+}} \phi(m)=0 .
$$

Proof. We have that (3.8) and (3.10) plainly follow from (3.5).

Now we prove $(3.9)$. For this, we use Proposition 3.1 and the fractional isoperimetric inequality in the form (3.7) to obtain that, if $|E|=m$,

$$
\mathcal{F}_{s, t}(E) \geqslant \frac{(1-t) P_{t}(E)}{2}-c_{0} m \geqslant \frac{m^{\frac{N-t}{N}}}{2 c_{N} t}-c_{0} m=\frac{m^{\frac{N-t}{N}}}{2 c_{N} t}\left(1-2 c_{0} c_{N} t m^{\frac{t}{N}}\right) .
$$

In particular, if $m$ is small enough, we have that

$$
\mathcal{F}_{s, t}(E) \geqslant \frac{m^{\frac{N-t}{N}}}{4 c_{N} t}
$$

and this implies $(3.9)$.

(proved

Lemma 3.6. Let $m_{1}$ be as in Lemma 3.5, and let $F$ be a minimizer of $\mathcal{F}_{s, t}$ among sets of measure $m>m_{1}$. We have

$$
\frac{c_{N}}{t} m^{\frac{N-t}{N}} \leqslant(1-t) P_{t}(F)<c_{0} m \text { and } \quad \frac{c_{N}}{t} m^{\frac{N-t}{N}}<s P_{s}(F) \leqslant c_{0} m,
$$

for some $c_{0}>0$.

Proof. By Lemma 3.5 we know that $(1-t) P_{t}(F)<s P_{s}(F)$, hence from (2.3) and from the fractional isoperimetric inequality (3.7) we get

$$
\frac{m^{\frac{N-t}{N}}}{c_{N} t} \leqslant(1-t) P_{t}(F)<s P_{s}(F) \leqslant c_{0}^{\frac{t-s}{t}} 2^{-\frac{s}{t}} m^{1-\frac{s}{t}}\left[(1-t) P_{t}(F)\right]^{\frac{s}{t}}
$$

with $c_{0}$ given in Proposition 3.1. Then $(1-t) P_{t}(F)<c_{0} 2^{-\frac{s}{t-s}} m$. This and $(2.3)$ also implies the desired bound on $s P_{s}(F)$.

Remark 3.7. By inspecting the proof of the Lemma 3.5 we obtain explicit estimates for $m_{0}$ and $m_{1}$ :

$$
\begin{aligned}
& m_{0} \geqslant\left[4 c_{0} c_{N} t\right]^{-\frac{N}{t}}=\left[4\left(\frac{c_{N} t 2^{s / t}}{t-s}\right)^{\frac{t}{t-s}} c_{N} t\right]^{-\frac{N}{t}} \\
& m_{1} \leqslant\left(\frac{c_{1}}{c_{2}}\right)^{\frac{N}{t-s}}=\left[\frac{(1-t) P_{t}(B)}{s P_{s}(B)}\right]^{\frac{N}{t-s}}|B| .
\end{aligned}
$$


Moreover, the first inequality in the second formula in 3.11

$$
\frac{c_{N}}{t}|F|^{\frac{N-t}{N}}<s P_{s}(F)
$$

entails that $|F| \rightarrow \infty$ as $t \rightarrow 0$ (and thus $\delta_{0} \rightarrow 0$ ). Indeed, letting $t=s+\delta_{0}$, and using the fact that $s P_{s}(F) \rightarrow N \omega_{N}|F|$ as $s \rightarrow 0$, after an elementary computation we get that

$$
m_{1} \geqslant|F| \geqslant\left(\frac{c_{N}}{s+\delta_{0}}\right)^{\frac{N}{s+\delta_{0}}} .
$$

which gives also a lower bound on $m_{1}$ in terms of $s$ and $\delta_{0}$. Notice that if $t \rightarrow 0$, then also $s$ and $\delta_{0}$ converge to 0 and so $m_{1} \rightarrow \infty$. Also it is not a direct consequence of our investigation, we stress that it is natural to expect that also if only $s$ converges to 0 , then $m_{1}$ diverges to $+\infty$.

We state an elementary numerical inequality which will be useful in the proof of the forthcoming Proposition 3.9 .

Lemma 3.8. Let $\gamma>0$ and $\lambda=(1+\gamma)^{1 / N}$. Then, for any $a, b \geqslant 0$, it holds

$$
\left(\lambda^{N-t}-1\right) a-\left(\lambda^{N-s}-1\right) b \leqslant \gamma(a-b) .
$$

Proof. To prove (3.12), we notice that

$$
\lim _{\gamma \rightarrow 0}(N-s)(1+\gamma)^{\frac{t-s}{N}}-(N-t)=t-s>0,
$$

hence we may take $\gamma$ small enough, such that

$$
(N-s)(1+\gamma)^{\frac{t-s}{N}}-(N-t) \geqslant \frac{t-s}{2} .
$$

So we write

$$
f(\gamma):=\left((1+\gamma)^{\frac{N-t}{N}}-1\right) a-\left((1+\gamma)^{\frac{N-s}{N}}-1\right) b=\left(\lambda^{N-t}-1\right) a-\left(\lambda^{N-s}-1\right) b,
$$

and we notice that $f(0)=0$ and

$$
\begin{aligned}
f^{\prime}(\gamma) & =\frac{N-t}{N}(1+\gamma)^{-\frac{t}{N}} a-\frac{N-s}{N}(1+\gamma)^{-\frac{s}{N}} b \\
& =\frac{N-t}{N}(1+\gamma)^{-\frac{t}{N}}(a-b)-\frac{b(1+\gamma)^{-\frac{t}{N}}}{N}\left[(N-s)(1+\gamma)^{\frac{t-s}{N}}-(N-t)\right] \\
& \leqslant(a-b)-\frac{b(1+\gamma)^{-\frac{t}{N}}(t-s)}{2 N},
\end{aligned}
$$

thanks to 3.13). In particular, $f^{\prime}(\gamma) \leqslant(a-b)$ and thus $f(\gamma) \leqslant \gamma(a-b)$, that establishes 3.12).

Proposition 3.9 (Non-optimality criterion). There exists $\varepsilon=\varepsilon\left(N, \delta_{0}\right)$ such that, if $F \subset \mathbb{R}^{N}$ can be written as $F=F_{1} \cup F_{2}$, with $\left|F_{1} \cap F_{2}\right|=0$,

$$
\begin{array}{ll} 
& \left|F_{2}\right| \leqslant \varepsilon \min \left(1,\left|F_{1}\right|\right), \\
\text { and } \quad(1-t)\left[P_{t}\left(F_{1}\right)+P_{t}\left(F_{2}\right)-P_{t}(F)\right] \leqslant \frac{\mathcal{F}_{s, t}\left(F_{2}\right)}{2},
\end{array}
$$

then there exists a set $G$ with $|G|=|F|$ and $\mathcal{F}_{s, t}(G)<\mathcal{F}_{s, t}(F)$ (i.e., $F$ is not a minimizer).

In addition, we have that the set $G$ is either a ball of volume $m$, or a dilation of the set $F_{1}$, according to the following formula:

$$
G=\sqrt[N]{1+\frac{\left|F_{2}\right|}{\left|F_{1}\right|}} F_{1}
$$


Proof. Let $m:=|F|, m_{1}:=\left|F_{1}\right|$ and $m_{2}:=\left|F_{2}\right|$. We may suppose that $\mathcal{F}_{s, t}(F)$ is less than or equal than $\mathcal{F}_{s, t}$ of the ball of volume $m, B_{m}$, otherwise we can take $G$ equal to such a ball, decrease the energy and finish our proof. That is, we may suppose that

$$
\mathcal{F}_{s, t}(F) \leqslant \mathcal{F}_{s, t}\left(B_{m}\right) \leqslant(1-t) P_{t}\left(B_{m}\right) \leqslant \frac{(1-t) P_{t}(B)}{|B|^{\frac{N-t}{N}}} m^{\frac{N-t}{N}} .
$$

Let $G=\lambda F_{1}$, with $\lambda:=\sqrt[N]{1+\gamma}$ and $\gamma=m_{2} / m_{1}$. Notice that this is in agreement with (3.16), and also $|G|=m$. Moreover, by (3.14) we have that

$$
\gamma \leqslant \frac{\varepsilon \min \left(1, m_{1}\right)}{m_{1}} \leqslant \varepsilon
$$

so that $\gamma \in(0,1)$ can be taken as small as we like.

By applying inequality (3.12) with $a=(1-t) P_{t}\left(F_{1}\right)$ and $b=s P_{s}\left(F_{1}\right)$, we obtain that

$$
\left(\lambda^{N-t}-1\right)(1-t) P_{t}\left(F_{1}\right)-\left(\lambda^{N-s}-1\right) s P_{s}\left(F_{1}\right) \leqslant \gamma\left[(1-t) P_{t}\left(F_{1}\right)-s P_{s}\left(F_{1}\right)\right] .
$$

As a consequence we get

$$
\begin{aligned}
\mathcal{F}_{s, t}(G) & =(1-t) P_{t}(G)-s P_{s}(G) \\
& =\lambda^{N-t}(1-t) P_{t}\left(F_{1}\right)-\lambda^{N-s} s P_{s}\left(F_{1}\right) \\
& =\mathcal{F}_{s, t}\left(F_{1}\right)+\left[\left(\lambda^{N-t}-1\right)(1-t) P_{t}\left(F_{1}\right)-\left(\lambda^{N-s}-1\right) s P_{s}\left(F_{1}\right)\right] \\
& \leqslant(1+\gamma) \mathcal{F}_{s, t}\left(F_{1}\right) .
\end{aligned}
$$

Thus we have, by 2.2 and 3.15 ,

$$
\begin{aligned}
\mathcal{F}_{s, t}(G)-\mathcal{F}_{s, t}(F) \leqslant & (1+\gamma) \mathcal{F}_{s, t}\left(F_{1}\right)-(1-t) P_{t}(F)+s P_{s}(F) \\
\leqslant & (1+\gamma) \mathcal{F}_{s, t}\left(F_{1}\right)-(1-t) P_{t}(F)+s P_{s}\left(F_{1}\right)+s P_{s}\left(F_{2}\right) \\
\leqslant & (1+\gamma) \mathcal{F}_{s, t}\left(F_{1}\right)+s P_{s}\left(F_{1}\right)+s P_{s}\left(F_{2}\right) \\
& +\frac{1}{2} \mathcal{F}_{s, t}\left(F_{2}\right)-(1-t) P_{t}\left(F_{1}\right)-(1-t) P_{t}\left(F_{2}\right) \\
= & \gamma \mathcal{F}_{s, t}\left(F_{1}\right)-\frac{1}{2} \mathcal{F}_{s, t}\left(F_{2}\right) .
\end{aligned}
$$

Furthermore by $(3.9)$, since $m_{2}$ can be chosen in $\left(0, m_{0}\right), m_{0}$ as in Lemma 3.5 (up to decreasing the value of $\varepsilon$ ), we have

$$
\mathcal{F}_{s, t}\left(F_{2}\right) \geqslant \phi\left(m_{2}\right) \geqslant \frac{c_{N}}{t} m_{2}^{\frac{N-t}{N}}
$$

Also, using again (2.2) and (3.15), we have that

$$
\begin{aligned}
\mathcal{F}_{s, t}\left(F_{1}\right) & =\left[(1-t) P_{t}\left(F_{1}\right)+(1-t) P_{t}\left(F_{2}\right)-s P_{s}\left(F_{1}\right)-s P_{s}\left(F_{2}\right)\right]-\mathcal{F}_{s, t}\left(F_{2}\right) \\
& \leqslant \mathcal{F}_{s, t}(F)+\left[(1-t) P_{t}\left(F_{1}\right)+(1-t) P_{t}\left(F_{2}\right)-(1-t) P_{t}(F)-\mathcal{F}_{s, t}\left(F_{2}\right)\right] \\
& \leqslant \mathcal{F}_{s, t}(F)-\frac{1}{2} \mathcal{F}_{s, t}\left(F_{2}\right)<\mathcal{F}_{s, t}(F) .
\end{aligned}
$$

This, 3.18) and (3.19) give that

$$
\mathcal{F}_{s, t}(G)-\mathcal{F}_{s, t}(F) \leqslant \gamma \mathcal{F}_{s, t}(F)-\frac{1}{2} \mathcal{F}_{s, t}\left(F_{2}\right) \leqslant \gamma \mathcal{F}_{s, t}(F)-\frac{c_{N}}{2 t} m_{2}^{\frac{N-t}{N}} .
$$


Accordingly, recalling (3.17) we conclude that

$$
\begin{aligned}
\mathcal{F}_{s, t}(G)-\mathcal{F}_{s, t}(F) & \leqslant \frac{(1-t) P_{t}(B)}{|B|^{\frac{N-t}{N}}} \gamma\left(m_{1}+m_{2}\right)^{\frac{N-t}{N}}-\frac{c_{N}}{2 t} m_{2}^{\frac{N-t}{N}} \\
& =m_{2}^{\frac{N-t}{N}}\left[\frac{(1-t) P_{t}(B)}{|B|^{\frac{N-t}{N}}} \gamma\left(\gamma^{-1}+1\right)^{\frac{N-t}{N}}-\frac{c_{N}}{2 t}\right] \\
& \leqslant m_{2}^{\frac{N-t}{N}}\left[\frac{(1-t) P_{t}(B)}{|B|^{\frac{N-t}{N}}} \gamma\left(2 \gamma^{-1}\right)^{\frac{N-t}{N}}-\frac{c_{N}}{2 t}\right] \\
& =m_{2}^{\frac{N-t}{N}}\left[\frac{2^{\frac{N-t}{N}}(1-t) P_{t}(B)}{|B|^{\frac{N-t}{N}}} \gamma^{\frac{t}{N}}-\frac{c_{N}}{2 t}\right]
\end{aligned}
$$

which is negative if $\gamma$ is small enough, i. e.

$$
\gamma<\left[\frac{c_{N}}{2 t} \frac{|B|^{\frac{N-t}{N}}}{2^{\frac{N-t}{N}}(1-t) P_{t}(B)}\right]^{\frac{N}{t}} .
$$

The proof is concluded.

When (3.15 does not hold, one obtains for free some interesting density bounds.

Given a measurable set $E$ we denote by $\partial^{m} E$ the measure theoretic boundary of $E$ defined as

$$
\partial^{m} E=\left\{x \in \mathbb{R}^{N}:\left|E \cap B_{r}(x)\right|>0 \text { and }\left|E \backslash B_{r}(x)\right|>0 \text { for all } r>0\right\} .
$$

Lemma 3.10. Let $F$ be a set of finite t-perimeter and volume $m$, and let $x_{0} \in \mathbb{R}^{N}$. Assume

$$
\begin{aligned}
& \text { either } F_{1}:=F \backslash B\left(x_{0}, r\right) \text { and } F_{2}:=F \cap B\left(x_{0}, r\right) \text {, } \\
& \text { or } F_{2}:=F \backslash B\left(x_{0}, r\right) \text { and } F_{1}:=F \cap B\left(x_{0}, r\right),
\end{aligned}
$$

and suppose that $\left|F_{2}\right|<m_{0}$, with $m_{0}$ be as in Lemma 3.5, and

$$
(1-t)\left[P_{t}\left(F_{1}\right)+P_{t}\left(F_{2}\right)-P_{t}(F)\right] \geqslant \frac{\mathcal{F}_{s, t}\left(F_{2}\right)}{2} .
$$

Then

$$
\int_{F_{1}} \int_{F_{2}} \frac{d x d y}{|x-y|^{N+t}} \geqslant \frac{c_{N}}{t(1-t)}\left|F_{2}\right|^{\frac{N-t}{N}}
$$

If $x_{0} \in \partial^{m} F$ and $(3.22)$ holds for any $r \leqslant r_{0}$, we also have the estimate

$$
\left|F \cap B\left(x_{0}, r\right)\right| \geqslant c_{0} r^{N} \quad \text { for all } r \in\left(0, r_{0}\right],
$$

where the constant $c_{0}>0$ depend only on $N$ and $\delta_{0}$.

Proof. Without loss of generality we can assume $x_{0}=0$. Also, using either (3.20) or (3.21), 3.22) and (2.1), we have that

$$
\int_{F_{1}} \int_{F_{2}} \frac{d x d y}{|x-y|^{N+t}}=\frac{1-t}{2(1-t)}\left(P_{t}\left(F_{1}\right)+P_{t}\left(F_{2}\right)-P_{t}(F)\right) \geqslant \frac{\mathcal{F}_{s, t}\left(F_{2}\right)}{4(1-t)} \geqslant \frac{\phi\left(\left|F_{2}\right|\right)}{4(1-t)} .
$$

This and (3.9) (which can be used here thanks to the fact that we are assuming $\left|F_{2}\right|<m_{0}$ ) imply 3.23 .

Now we prove (3.24). For this, we take $F_{1}$ and $F_{2}$ as in 3.20 and we define $\mu(r):=\mid B(0, r) \cap$ $F|=| F_{2} \mid$. Note that by the co-area formula

$$
\mu^{\prime}(r)=\mathcal{H}^{N-1}(\partial B(0, r) \cap F), \quad \text { for a. e. } r .
$$


Then, by (3.23) and the fact that $F_{1}:=F \backslash B(0, r) \subset(B(0, r))^{c}$,

$$
\frac{c_{N}}{t(1-t)} \mu(r)^{\frac{N-t}{N}} \leqslant \int_{F_{2}} \int_{F_{1}} \frac{d x d y}{|x-y|^{N+t}} \leqslant \int_{F_{2}} \int_{(B(0, r))^{c}} \frac{d x d y}{|x-y|^{N+t}} .
$$

For any $x \in F \cap B(0, r)$, we have

$$
\int_{(B(0, r))^{c}} \frac{d y}{|x-y|^{N+t}} \leqslant \int_{(B(x, r-|x|))^{c}} \frac{d y}{|x-y|^{N+t}}=\frac{N \omega_{N}}{t}(r-|x|)^{-t}
$$

that leads to

$$
\int_{F_{2}} \int_{(B(0, r))^{c}} \frac{d x d y}{|x-y|^{N+t}} \leqslant \frac{c_{N}}{t} \int_{0}^{r} \mu^{\prime}(z)(r-z)^{-t} d z .
$$

Finally we arrive at the following integro-differential inequality

$$
\mu(r)^{\frac{N-t}{N}} \leqslant c_{N}(1-t) \int_{0}^{r} \mu^{\prime}(z)(r-z)^{-t} d z .
$$

We may integrate the last inequality in the $r$ variable on the interval $(0, \rho)$ and get

$$
\int_{0}^{\rho} \mu(r)^{\frac{N-t}{N}} d r \leqslant c_{N}(1-t) \int_{0}^{\rho} \int_{0}^{r} \mu^{\prime}(z)(r-z)^{-t} d z d r
$$

interchanging the order of integration,

$$
\int_{0}^{\rho} \int_{0}^{r} \mu^{\prime}(z)(r-z)^{-t} d z d r=\int_{0}^{\rho} \mu^{\prime}(z) \int_{z}^{\rho}(r-z)^{-t} d r d z
$$

we get

$$
\int_{0}^{\rho} \mu(r)^{\frac{N-t}{N}} d r \leqslant c_{N} \rho^{1-t} \mu(\rho)
$$

Now we arrive at the desired result, indeed, following [12] (see the end of p. 9), it is possible to prove that

$$
\mu(r) \geqslant g(r):=\left[\frac{1}{2 c_{N}(N+1-t)}\right]^{\frac{N}{t}} r^{N}
$$

for any $r<r_{0}=\left(m_{0} / \omega_{N}\right)^{1 / N}$, where $g$ satisfies

$$
\int_{0}^{\rho} g(r)^{\frac{N-t}{N}} d r \geqslant 2 c_{N} \rho^{1-t} g(\rho)
$$

with the same constant $c_{N}$ as in (3.25).

The combination of Proposition 3.9 and Lemma 3.10 yield the following density estimate:

Proposition 3.11. There exist $r_{0}=r_{0}\left(m, N, \delta_{0}\right)>0$ such that, if $F$ is a minimizer for $\phi(m)$ and $x_{0} \in \partial^{m} F$, there holds

$$
\left|B\left(x_{0}, r\right) \cap F\right| \geqslant c_{0} r^{N}
$$

for any $r<r_{0}$, where $c_{0}$ is as in (3.24).

Proof. Let $F_{1}$ and $F_{2}$ be as in 3.20 . Up to choosing $r_{0}$ small enough, that is,

$$
\omega_{N} r_{0}^{N} \leqslant \varepsilon\left(N, \delta_{0}\right) \min (1, m),
$$

we can suppose that $F_{1}$ and $F_{2}$ satisfy the hypotheses of Proposition 3.9. Thus, since $F$ is a minimum, we obtain that 3.15 cannot hold true. Hence 3.22 is satisfied, and so we can apply (3.24) in Lemma 3.10 and obtain the desired result. 
Proposition 3.12. Let $F$ be a minimum for $\phi(m)$. Then $F$ is essentially bounded. Moreover, if $t=1$, for any $s<t, s \in(0,1), F$ is also essentially connected in the sense of [2], that is, it cannot be decomposed into two disjoint sets $F_{1}$ and $F_{2}$ of positive measure such that $P(F)=P\left(F_{1}\right)+P\left(F_{2}\right)$.

Proof. Let $F$ be a minimum. First we prove that it is bounded. By contradiction, if not, there exists a sequence $x_{k} \in \partial^{m} F$ such that $\left|x_{k}\right| \rightarrow \infty$ as $k \rightarrow \infty$. In particular, up to a subsequence, we may suppose that all the balls $B\left(x_{k}, 1\right)$ are disjoint, hence so are the balls $B\left(x_{k}, r\right)$ when $r \in$ $(0,1)$. Hence

$$
m=|F| \geqslant \sum_{k}\left|B\left(x_{k}, r\right) \cap F\right| .
$$

On the other hand, by Proposition 3.11, we know that $\left|B\left(x_{k}, r\right) \cap F\right| \geqslant c_{0} r^{N}$ if $r$ is small enough, hence we obtain that

$$
m \geqslant \sum_{k} c_{0} r^{N}=+\infty
$$

which is clearly not possible.

This proves that $F$ is bounded. Now we show that, if $t=1, F$ is also essentially connected. Suppose, by contradiction, that $F$ can be decomposed into two disjoint sets $F_{1}$ and $F_{2}$ of positive measure such that

$$
P(F)=P\left(F_{1}\right)+P\left(F_{2}\right) .
$$

Since $F$ is bounded, so are $F_{1}$ and $F_{2}$, say $F_{1}, F_{2} \subseteq B(0, R)$, for some $R>0$. Hence, we consider the translation $F_{2, k}:=F_{2}+(k, 0, \ldots, 0)$ and we observe that if $x \in F_{1}$ and $y \in F_{2, k}$ we have that

$$
|x-y| \geqslant|y|-|x| \geqslant k-2 R \geqslant \frac{k}{2}
$$

if $k$ is large enough. Accordingly, we have that

$$
\int_{F_{1}} \int_{F_{2, k}} \frac{d x d y}{|x-y|^{N+s}} \leqslant \int_{B(0, R)} \int_{B(0, R)+(k, 0, \ldots, 0)} \frac{d x d y}{(k / 2)^{N+s}}=\frac{c_{N} R^{2 N}}{k^{N+s}}
$$

and so

$$
\lim _{k \rightarrow+\infty} \int_{F_{1}} \int_{F_{2, k}} \frac{d x d y}{|x-y|^{N+s}}=0 .
$$

Notice also that, if $G_{k}:=F_{1} \cup F_{2, k}$ we have that $\left|G_{k}\right|=\left|F_{1}\right|+\left|F_{2, k}\right|=\left|F_{1}\right|+\left|F_{2}\right|=|F|$, for $k$ large, and so, by the minimality of $F,(2.1),(2.2)$ and $(3.26)$ we have that

$$
\begin{aligned}
& N \omega_{N} P\left(F_{1}\right)+N \omega_{N} P\left(F_{2}\right)-s P_{s}\left(F_{1}\right)-s P_{s}\left(F_{2}\right)+2 s \int_{F_{1}} \int_{F_{2}} \frac{d x d y}{|x-y|^{N+s}} \\
= & N \omega_{N} P(F)-s P_{s}(F) \\
= & \mathcal{F}_{s, 1}(F) \\
\leqslant & \mathcal{F}_{s, 1}\left(G_{k}\right) \\
= & N \omega_{N} P\left(G_{k}\right)-s P_{s}\left(G_{k}\right) \\
\leqslant & N \omega_{N} P\left(F_{1}\right)+N \omega_{N} P\left(F_{2, k}\right)-s P_{s}\left(F_{1}\right)-s P_{s}\left(F_{2, k}\right)+2 s \int_{F_{1}} \int_{F_{2, k}} \frac{d x d y}{|x-y|^{N+s}} \\
= & N \omega_{N} P\left(F_{1}\right)+N \omega_{N} P\left(F_{2}\right)-s P_{s}\left(F_{1}\right)-s P_{s}\left(F_{2}\right)+2 s \int_{F_{1}} \int_{F_{2, k}} \frac{d x d y}{|x-y|^{N+s}} .
\end{aligned}
$$


Therefore, taking the limit as $k \rightarrow+\infty$ and using (3.27), we obtain that

$$
2 s \int_{F_{1}} \int_{F_{2}} \frac{d x d y}{|x-y|^{N+s}} \leqslant 0 .
$$

This says that either $F_{1}$ or $F_{2}$ must have zero measure, against our assumptions.

We conclude the section with the following estimate on the fractional isoperimetric deficit, which will be important to localize minimizing sequences.

Lemma 3.13. There exists $m_{2}=m_{2}\left(N, \delta_{0}\right)$ such that for any $m \in\left(0, m_{2}\right)$ the following statement holds true.

Let $F \subset \mathbb{R}^{N}$ be a set of finite perimeter. Assume that $\mathcal{F}_{s, t}(F) \leqslant \mathcal{F}_{s, t}\left(B_{m}\right)$. Then there exists $c_{0}>0$ such that

$$
\delta P_{t}(F)=\frac{P_{t}(F)-P_{t}\left(B_{m}\right)}{P_{t}\left(B_{m}\right)} \leqslant c_{0} m^{\frac{t-s}{N}} .
$$

In addition, there exists a translation of $F$ (still denoted by $F$ for simplicity) such that

$$
\left|F \Delta B_{m}\right| \leqslant c_{0} m^{1+\frac{t-s}{2 N}} .
$$

Proof. First recall that

$$
P_{t}\left(B_{m}\right)=\frac{P_{t}(B)}{|B|^{\frac{N-t}{N}}} m^{\frac{N-t}{N}}
$$

Also, by our assumptions,

$$
(1-t) P_{t}(F)-s P_{s}(F)=\mathcal{F}_{s, t}(F) \leqslant \mathcal{F}_{s, t}\left(B_{m}\right) \leqslant(1-t) P_{t}\left(B_{m}\right) .
$$

Using (3.4) we have that

$$
\begin{aligned}
s P_{s}(F) & \leqslant c_{0}^{1-\frac{s}{t}} m^{1-\frac{s}{t}}\left[(1-t) P_{t}\left(B_{m}\right)+c_{0} m\right]^{\frac{s}{t}} \\
& =c_{0}^{1-\frac{s}{t}} m^{1-\frac{s}{t}}\left[\frac{(1-t) P_{t}(B)}{|B|^{\frac{N-t}{N}}} m^{\frac{N-t}{N}}+c_{0} m\right]^{\frac{s}{t}} \\
& \leqslant c_{0}^{1-\frac{s}{t}}\left[\frac{(1-t) P_{t}(B)}{|B|^{\frac{N-t}{N}}}+c_{0}\right]^{\frac{s}{t}} m^{\frac{N-s}{N}},
\end{aligned}
$$

for small $m$. From this and (3.31), we have that

$$
\frac{P_{t}(F)-P_{t}\left(B_{m}\right)}{P_{t}\left(B_{m}\right)} \leqslant c_{0}^{1-\frac{s}{t}}\left[\frac{(1-t) P_{t}(B)}{|B|^{\frac{N-t}{N}}}+c_{0}\right]^{\frac{s}{t}} \frac{|B|^{\frac{N-t}{N}}}{(1-t) P_{t}(B)} m^{\frac{N-s}{N}-\frac{N-t}{N}} \leqslant c_{0} m^{\frac{t-s}{N}} .
$$

This proves 3.28 .

To prove $(3.29)$ it is sufficient to use (3.28) and the estimate

$$
c_{0} \delta P_{t}(F) \geqslant \frac{\left|F \Delta B_{m}\right|^{2}}{\left|B_{m}\right|^{2}},
$$

which was proved in [18, Theorem 1.1] for any $t \geqslant \delta_{0}>0$. Together with (3.28) and possibly increasing the constant $c_{0}$, this implies 3.29. 


\section{Existence OF Minimizers}

In order to prove the first statement in Theorem 1.2 , and for further use as well, we prove a general result on integro-differential equations:

Lemma 4.1. Let $m, t \in(0,1)$. Let $c, \bar{\rho} \geqslant 0$ be such that

$$
c \geqslant(1-t) m^{\frac{t}{N}},
$$

and let $\mu:[0,+\infty) \rightarrow[0, m]$ be a non-increasing function such that

$$
-\int_{\rho}^{\infty} \mu^{\prime}(z)(z-\rho)^{-t} d z \geqslant \frac{3 c}{1-t} \mu(\rho)^{\frac{N-t}{N}} \quad \text { for all } \rho \geqslant \bar{\rho} .
$$

Then, there holds

$$
\mu\left(\bar{\rho}+\frac{(2 m)^{\frac{t}{N}} N}{c t}\right)=0 .
$$

Proof. Integrating (4.2) between $R \geqslant \bar{\rho}$ and $+\infty$, we obtain

$$
-\int_{R}^{\infty}\left(\int_{\rho}^{\infty} \mu^{\prime}(z)(z-\rho)^{-t} d z\right) d \rho \geqslant \frac{3 c}{1-t} \int_{R}^{\infty} \mu(\rho)^{\frac{N-t}{N}} d \rho .
$$

Also, if $z \in[R, R+1]$ we have that $z-R \leqslant 1$ and so, since $\mu^{\prime} \leqslant 0$ a. e., we get that

$$
-\int_{R}^{R+1} \mu^{\prime}(z)(z-R)^{1-t} d z \leqslant-\int_{R}^{R+1} \mu^{\prime}(z) d z=\mu(R)-\mu(R+1) .
$$

Therefore, interchanging the order of integration in (4.4), integrating by parts and using that $\mu \in[0, m]$ and $(4.1)$, we see that

$$
\begin{aligned}
-\int_{R}^{\infty}\left(\int_{\rho}^{\infty} \mu^{\prime}(z)(z-\rho)^{-t} d z\right) d \rho & =-\int_{R}^{\infty}\left(\int_{R}^{z} \mu^{\prime}(z)(z-\rho)^{-t} d \rho\right) d z \\
& =-\frac{1}{1-t} \int_{R}^{\infty} \mu^{\prime}(z)(z-R)^{1-t} d z \\
& \leqslant \frac{\mu(R)-\mu(R+1)}{1-t}-\frac{1}{1-t} \int_{R+1}^{\infty} \mu^{\prime}(z)(z-R)^{1-t} d z \\
& =\frac{\mu(R)}{1-t}+\int_{R+1}^{\infty} \mu(z)(z-R)^{-t} d z \\
& \leqslant \frac{\mu(R)}{1-t}+\int_{R+1}^{\infty} \mu(z) d z \\
& \leqslant \frac{\mu(R)}{1-t}+m^{\frac{t}{N}} \int_{R}^{\infty} \mu(z)^{\frac{N-t}{N}} d z \\
& \leqslant \frac{1}{1-t}\left(\mu(R)+c \int_{R}^{\infty} \mu(z)^{\frac{N-t}{N}} d z\right) .
\end{aligned}
$$

Recalling (4.4), this gives the integro-differential inequality

$$
\mu(\rho) \geqslant 2 c \int_{\rho}^{\infty} \mu(z)^{\frac{N-t}{N}} d z \quad \text { for all } \rho \geqslant \bar{\rho} .
$$


Let now

$$
g(\rho):= \begin{cases}{\left[(2 \mu(\bar{\rho}))^{\frac{t}{N}}-\frac{c t}{N}(\rho-\bar{\rho})\right]^{\frac{N}{t}}} & \text { if } \rho \in\left[\bar{\rho}, \bar{\rho}+\frac{(2 \mu(\bar{\rho}))^{\frac{t}{N}} N}{c t}\right] \\ 0 & \text { if } \rho>\bar{\rho}+\frac{(2 \mu(\bar{\rho}))^{\frac{t}{N}} N}{c t} .\end{cases}
$$

Notice that $g$ is continuous and it satisfies

$$
2 c \int_{\rho}^{\infty} g(z)^{\frac{N-t}{N}} d z=2 g(\rho) \quad \text { for all } \rho \in\left[\bar{\rho}, \bar{\rho}+\frac{(2 \mu(\bar{\rho}))^{\frac{t}{N}} N}{c t}\right] .
$$

We now claim that

$$
g(\rho) \geqslant \mu(\rho) \quad \text { for all } \rho \in\left[\bar{\rho}, \bar{\rho}+\frac{(2 \mu(\bar{\rho}))^{\frac{t}{N}} N}{c t}\right] .
$$

Indeed, we consider the set $I:=\{\rho>\bar{\rho}: \mu(z) \geqslant g(z)$ for all $z \geqslant \rho\}$. By construction, $I \subseteq[\bar{\rho},+\infty)$. Furthermore, if $z \geqslant \bar{\rho}+\left[(2 \mu(\bar{\rho}))^{\frac{t}{N}} N\right] / c t$ then $g(z)=0 \leqslant \mu(z)$, therefore $\bar{\rho}+$ $\left[(2 \mu(\bar{\rho}))^{\frac{t}{N}} N\right] /$ ct $\in I$. As a consequence, we can define $R_{*}:=\inf I$, and we have that

$$
R_{*} \in\left[\bar{\rho}, \bar{\rho}+\left[(2 \mu(\bar{\rho}))^{\frac{t}{N}} N\right] / c t\right] .
$$

By definition of $R_{*}$, there exists a sequence $R_{n} \rightarrow R_{*}$, with $R_{n} \leqslant R_{*}$, such that $g\left(R_{n}\right)>\mu\left(R_{n}\right)$. Then, recalling (4.5) and (4.6), we have

$$
\begin{aligned}
g\left(R_{n}\right) & >\mu\left(R_{n}\right) \\
& \geqslant 2 c \int_{R_{n}}^{\infty} \mu(z)^{\frac{N-t}{N}} d z \\
& \geqslant 2 c \int_{R_{n}}^{R_{*}} \mu(z)^{\frac{N-t}{N}} d z+2 c \int_{R_{*}}^{\infty} g(z)^{\frac{N-t}{N}} d z \\
& =2 c \int_{R_{n}}^{R_{*}} \mu(z)^{\frac{N-t}{N}} d z+2 g\left(R_{*}\right) .
\end{aligned}
$$

Passing to the limit in 4.9 as $n \rightarrow+\infty$ we get $g\left(R_{*}\right) \geqslant 2 g\left(R_{*}\right)$, which means $g\left(R_{*}\right)=0$. This implies that $R_{*} \geqslant \bar{\rho}+\left[(2 \mu(\bar{\rho}))^{\frac{t}{N}} N\right] /$ ct.

This information, combined with (4.8), gives that $R_{*}=\bar{\rho}+\left[(2 \mu(\bar{\rho}))^{\frac{t}{N}} N\right] / c t$, and this in turn implies 4.7.

Then, we evaluate (4.7) at $\rho=\bar{\rho}+\left[(2 \mu(\bar{\rho}))^{\frac{t}{N}} N\right] /$ ct and we obtain 4.3.

With the above result, we are able to prove the first statement in Theorem 1.2 , concerning the existence of minimizers for small volumes.

Theorem 4.2. For any $0 \leqslant s<t \leqslant 1, t-s \geqslant \delta_{0}>0$, there exists $\bar{m}_{0}=\bar{m}_{0}\left(N, \delta_{0}\right)>0$ such that for all $m \in\left(0, \bar{m}_{0}\right)$, problem 1.7 has a minimizer $F \subset \mathbb{R}^{N}$.

Proof. Suppose $0<s<t<1$. We use the Direct Method of the Calculus of Variations. Let us consider a minimizing sequence $\left\{F_{k}\right\} \subset \mathbb{R}^{N}$, that is a sequence of sets of finite $t$-perimeter $F_{k}$ with $\left|F_{k}\right|=m$ such that

$$
\lim _{k \rightarrow \infty} \mathcal{F}_{s, t}\left(F_{k}\right)=\phi(m)
$$


Let also set $r_{m}:=\left(m / \omega_{N}\right)^{1 / N}>0$, so that $\left|B\left(0, r_{m}\right)\right|=m$. Our goal is to show that we can reduce ourselves to the case in which $F_{k}$ lies in a large ball, independent of $k$. More precisely, we claim that there exist $\rho_{*}>0$ and sets $G_{k}$, with $\left|G_{k}\right|=m$, such that

$$
G_{k} \subseteq B\left(0, \rho_{*}\right) \text { and } \mathcal{F}_{s, t}\left(G_{k}\right) \leqslant \mathcal{F}_{s, t}\left(F_{k}\right) .
$$

To prove it, we take $\rho \geqslant r_{m}$ and we set

$$
X_{k}^{\rho}:=F_{k} \cap B(0, \rho) \text { and } Y_{k}^{\rho}:=F_{k} \backslash B(0, \rho) .
$$

We distinguish two cases:

$$
\begin{array}{ll}
\text { (4.13) either for any } \rho \geqslant r_{m} \text { we have } & (1-t)\left[P_{t}\left(X_{k}^{\rho}\right)+P_{t}\left(Y_{k}^{\rho}\right)-P_{t}\left(F_{k}\right)\right] \geqslant \frac{\mathcal{F}_{s, t}\left(Y_{k}^{\rho}\right)}{2} \\
\text { (4.14) or there exists } \rho \geqslant r_{m} \text { such that } & (1-t)\left[P_{t}\left(X_{k}^{\rho}\right)+P_{t}\left(Y_{k}^{\rho}\right)-P_{t}\left(F_{k}\right)\right] \leqslant \frac{\mathcal{F}_{s, t}\left(Y_{k}^{\rho}\right)}{2} .
\end{array}
$$

Let us first deal with (4.13). In this case we can apply Lemma 3.10 using the setting in (3.21): accordingly, from $(3.23)$ we see that

$$
\int_{X_{k}^{\rho}} \int_{Y_{k}^{\rho}} \frac{d x d y}{|x-y|^{N+t}} \geqslant \frac{c_{N}}{t(1-t)}\left|Y_{k}^{\rho}\right|^{\frac{N-t}{N}} .
$$

Let us define the non-increasing function $\eta(\rho):=\left|F_{k} \backslash B(0, \rho)\right|=\left|Y_{k}^{\rho}\right|$. Note that by the co-area formula

$$
\eta^{\prime}(\rho)=-\mathcal{H}^{N-1}(\partial B(0, \rho) \cap F), \quad \text { for a. e. } \rho>0 .
$$

Proceeding as in the proof of Lemma 3.10 , we have

$$
\begin{aligned}
\int_{Y_{k}^{\rho}} \int_{X_{k}^{\rho}} \frac{d x d y}{|x-y|^{N+t}} & \leqslant \int_{Y_{k}^{\rho}} \int_{B(0, \rho)} \frac{d x d y}{|x-y|^{N+t}} \\
& \leqslant \int_{Y_{k}^{\rho}}\left(\int_{(B(y,|y|-\rho))^{c}} \frac{d x}{|x-y|^{N+t}}\right) d y \\
& \leqslant-\frac{N \omega_{N}}{t} \int_{\rho}^{\infty} \eta^{\prime}(z)(z-\rho)^{-t} d z
\end{aligned}
$$

whence

$$
-\int_{\rho}^{\infty} \eta^{\prime}(z)(z-\rho)^{-t} d z \geqslant \frac{c_{N}}{1-t} \eta(\rho)^{\frac{N-t}{N}}
$$

that is, $\eta$ satisfies inequality 4.2 . We now apply Lemma 4.1 with $\mu=\eta, c=c_{N} / 3$ and $\bar{\rho}=r_{m}$. Notice that, possibly reducing $\bar{m}_{0}$, we can ensure that condition (4.1) is satisfied. From (4.3) we conclude that

$$
\eta\left(r_{m}+\frac{3(2 m)^{\frac{t}{N}} N}{c_{N} t}\right)=0,
$$

that is,

$$
F_{k} \subseteq B\left(0, r_{m}+\frac{3(2 m)^{\frac{t}{N}} N}{c_{N} t}\right) .
$$

This proves 4.11 with $\rho_{*}$ given by

$$
\rho_{*}:=r_{m}+\frac{3(2 m)^{\frac{t}{N}} N}{c_{N} t}
$$

in the case where 4.13 holds (here one can take $G_{k}:=F_{k}$ ). 
We now deal with case (4.14). In this case, we use (3.29) and we obtain (up to a translation of $F_{k}$ that is still denoted by $F_{k}$ ) that

$$
\left|F_{k} \backslash B\left(0, r_{m}\right)\right|+\left|B\left(0, r_{m}\right) \backslash F_{k}\right|=\left|F_{k} \Delta B\left(0, r_{m}\right)\right| \leqslant c_{0} m^{1+\frac{t-s}{2 N}},
$$

$c_{0}$ as in Lemma 3.13. In particular, if $\rho \geqslant r_{m}$ is the one given by (4.14) we have that

$$
\begin{aligned}
\left|F_{k} \cap B(0, \rho)\right| & \geqslant\left|F_{k} \cap B\left(0, r_{m}\right)\right| \\
& =\left|B\left(0, r_{m}\right)\right|-\left|B\left(0, r_{m}\right) \backslash F_{k}\right| \\
& \geqslant m-c_{0} m^{1+\frac{t-s}{2 N}} \\
& \geqslant \frac{m}{2}
\end{aligned}
$$

if $m$ is small enough, i. e.

$$
m \leqslant\left[\frac{1}{2 c_{0}}\right]^{\frac{2 N}{t-s}}
$$

and moreover

$$
\left|F_{k} \backslash B(0, \rho)\right| \leqslant\left|F_{k} \backslash B\left(0, r_{m}\right)\right| \leqslant c_{0} m^{1+\frac{t-s}{2 N}} .
$$

Therefore, for small $m$, recalling $(4.12)$ we see that

$$
\begin{gathered}
2 c_{0} m^{\frac{t-s}{2 N}} \min \left(1,\left|X_{k}^{\rho}\right|\right)=2 c_{0} m^{\frac{t-s}{2 N}} \min \left(1,\left|F_{k} \cap B(0, \rho)\right|\right) \geqslant 2 c_{0} m^{\frac{t-s}{2 N}} \frac{m}{2} \\
=c_{0} m^{1+\frac{t-s}{2 N}} \geqslant\left|F_{k} \backslash B(0, \rho)\right|=\left|Y_{k}^{\rho}\right| .
\end{gathered}
$$

Thanks to this and (4.14), we can apply Proposition 3.9, with $\varepsilon:=2 c_{0} m^{\frac{t-s}{2 N}}, F_{1}:=X_{k}^{\rho}$ and $F_{2}:=Y_{k}^{\rho}$.

Hence, from Proposition 3.9 , we find $G_{k}$ such that $\mathcal{F}_{s, t}\left(G_{k}\right) \leqslant \mathcal{F}_{s, t}\left(F_{k}\right)$; notice also that, in light of (3.16), we know that $G_{k}$ is either a ball or a dilation of $X_{k}^{\rho}$, which is contained in $B(0,2 \rho)$. Thus also $G_{k}$ is contained in a ball of universal radius, and this establishes (4.11) also in case 4.14 .

Thus, by (4.11), we have constructed a minimizing sequence $G_{k}$ that is uniformly contained in a fixed ball. By Proposition 3.1. we also obtain that

$$
(1-t) P_{t}\left(G_{k}\right) \leqslant 2\left[\mathcal{F}_{s, t}\left(G_{k}\right)+c_{0} m\right] \leqslant 2\left[\mathcal{F}_{s, t}\left(B\left(0, r_{m}\right)\right)+c_{0} m\right]
$$

hence the $t$-perimeter of $G_{k}$ is bounded uniformly in $k$.

By the compact embedding of $H^{\frac{t}{2}}$ into $H^{\frac{s}{2}}$ (see [16, Section 7]), up to extracting a subsequence, the sets $G_{k}$ converge in $W^{s, 1}$ (hence also in $L^{1}$ ) to a limit set $G$, and it holds

$$
\lim _{k \rightarrow+\infty} P_{s}\left(G_{k}\right)=P_{s}(G) .
$$

The lower semicontinuity of the $t$-perimeter yields that

$$
\liminf _{k \rightarrow+\infty} P_{t}\left(G_{k}\right) \geqslant P_{t}(G)
$$

Hence, by (4.10) and 4.11,

$$
\begin{aligned}
\mathcal{F}_{s, t}(G) & =(1-t) P_{t}(G)-s P_{s}(G) \leqslant \liminf _{k \rightarrow+\infty}\left[(1-t) P_{t}\left(G_{k}\right)-s P_{s}\left(G_{k}\right)\right] \\
& =\liminf _{k \rightarrow+\infty} \mathcal{F}_{s, t}\left(G_{k}\right) \leqslant \liminf _{k \rightarrow+\infty} \mathcal{F}_{s, t}\left(F_{k}\right) \leqslant \phi(m),
\end{aligned}
$$

hence $\mathcal{F}_{s, t}(G)=\phi(m)$ and so $F:=G$ is the desired minimizer. 
In the case $0=s<t \leqslant 1$, our problem reduces to the (fractional) isoperimetric problem, hence it is well known that there exists a minimizer $F$ for 1.7$)$ and it is a ball of volume $m$, for any $m>0$.

When $0<s<t=1$ the previous arguments can be easily adapted, including the analog of Lemma 4.1 which becomes an ordinary differential inequality, and the only difference is that one needs to use the compact embedding of $B V$ into $H^{\frac{s}{2}}$ for $0<s<1$.

\section{REgularity OF MINIMIZERS}

The aim of this section is to prove the regularity and rigidity theory necessary to prove the second statement in Theorem 1.2 and Theorem 1.3 . We begin with a simple observation.

Lemma 5.1. Let $\phi$ be the function describing problem (1.7). Then $F$ is a minimizer of $\phi(m)$ if and only if $\mathrm{F} / \mathrm{m}^{1 / N}$ is a minimizer of problem

$$
\min \left\{(1-t) P_{t}(U)-m^{\frac{t-s}{N}} s P_{s}(U):|U|=1\right\} .
$$

Proof. Let $F \subseteq \mathbb{R}^{N}$ such that $|F|=m$ and let $U=F / m^{1 / N}$. Then

$$
\begin{aligned}
(1-t) P_{t}(F)-s P_{s}(F) & =(1-t) P_{t}\left(m^{1 / N} U\right)-s P_{s}\left(m^{1 / N} U\right) \\
& =m^{\frac{N-t}{N}}\left[(1-t) P_{t}(U)-m^{\frac{t-s}{N}} s P_{s}(U)\right],
\end{aligned}
$$

which gives the desired result.

The previous lemma allows us to consider, in what follows, the functional

$$
\mathcal{F}_{s, t}^{\varepsilon}=(1-t) P_{t}-\varepsilon s P_{s},
$$

where we set $\varepsilon=m^{(t-s) / N}$. Indeed, the behavior of a minimizer of $\phi(m)$ is the same, up to a rescaling, to that of

$$
\min \left\{\mathcal{F}_{s, t}^{\varepsilon}(E):|E|=\omega_{N}\right\}
$$

Indeed

$F$ is a minimizer for problem (5.1) if and only if

$$
\left(\frac{m}{\omega_{N}}\right)^{\frac{1}{N}} F \text { is a minimizer for problem } 1.7 \text { with } \varepsilon=\left(\frac{m}{\omega_{N}}\right)^{\frac{t-s}{N}} \text {. }
$$

The next lemma allows us to say that if $F$ is a set of $\mathbb{R}^{N}$ such that ||$F\left|-\omega_{N}\right|$ is small enough than the volume constraint can be dropped. Let us consider the following problem:

$$
\min \left\{\mathcal{G}_{\varepsilon, \Lambda}(E):|| E\left|-\omega_{N}\right|<\Lambda^{-1}\right\},
$$

for some $\Lambda>0$, where

$$
\mathcal{G}_{\varepsilon, \Lambda}(E)=(1-t) P_{t}(E)-\varepsilon s P_{s}(E)+\Lambda|| E\left|-\omega_{N}\right| .
$$

Letting

$$
\varepsilon_{0}:=\left(\frac{\bar{m}_{0}}{\omega_{N}}\right)^{\frac{t-s}{N}},
$$

with $\bar{m}_{0}$ as in Theorem 4.2 , we have the following result:

Lemma 5.2. There exists $\Lambda_{0}=\Lambda_{0}\left(N, \delta_{0}\right)>0$ such that $F_{\varepsilon}$ is a volume constrained minimizer of problem (5.1), with $\varepsilon<\varepsilon_{0}$, if and only if $F_{\varepsilon}$ is a minimizer of problem (5.3), for any $\Lambda \geqslant \Lambda_{0}\left(1+\varepsilon_{0}\right)$. 
Proof. First, let $F_{\varepsilon}$ be a minimizer of problem (5.3) with $\left|F_{\varepsilon}\right|=\omega_{N}$. Then, for any set $G$ with $|G|=\omega_{N}$, we have that

$$
\mathcal{F}_{s, t}^{\varepsilon}(G)=\mathcal{G}_{\varepsilon, \Lambda}(G) \geqslant \mathcal{G}_{\varepsilon, \Lambda}\left(F_{\varepsilon}\right)=\mathcal{F}_{s, t}^{\varepsilon}\left(F_{\varepsilon}\right)
$$

which shows that $F_{\varepsilon}$ is a minimizer of problem (5.1).

Viceversa, we prove that a volume constrained minimizer $F_{\varepsilon}$ of problem (5.1), with $\varepsilon<\varepsilon_{0}$, is also a minimizer of $(5.3)$ for any $\Lambda$ sufficiently large. For this, we argue by contradiction and we assume that there exist a sequence $\Lambda_{n} \rightarrow+\infty$, and sets $E_{n} \subset \mathbb{R}^{N}$ such that, letting $\mathcal{G}_{n}:=\mathcal{G}_{\varepsilon, \Lambda_{n}}$, we have

$$
\mathcal{G}_{n}\left(E_{n}\right)<\mathcal{G}_{n}\left(F_{\varepsilon}\right)=\mathcal{F}_{s, t}^{\varepsilon}\left(F_{\varepsilon}\right) .
$$

Notice that for all $n \in \mathbb{N}$ there holds

$$
\sigma_{n}:=|| E_{n}\left|-\omega_{N}\right|>0 .
$$

Indeed, if by contradiction we suppose that $\sigma_{n}=0$ for some $n \in \mathbb{N}$, we would have that $\left|E_{n}\right|=\omega_{N}$, thus

$$
\mathcal{G}_{n}\left(E_{n}\right)=\mathcal{F}_{s, t}^{\varepsilon}\left(E_{n}\right) \geqslant \mathcal{F}_{s, t}^{\varepsilon}\left(F_{\varepsilon}\right)
$$

due to the minimality of $F_{\varepsilon}$. This would be in contradiction with (5.5), and so (5.6) is proved. We also claim that there exists a constant $c_{0}>0$ independent of $n$, such that

$$
(1-t) P_{t}\left(E_{n}\right) \leqslant c_{0} \quad \text { and } \quad s P_{s}\left(E_{n}\right) \leqslant c_{0} \quad \text { for all } n \in \mathbb{N} .
$$

To show this, proceeding as in Proposition 3.1 and thanks to 5.5 , we see that, for $\Lambda_{n} \geqslant c_{0} \varepsilon_{0}^{\frac{t}{t-s}}$, we have

$$
\begin{aligned}
(1-t) P_{t}\left(E_{n}\right) & \leqslant 2\left[\mathcal{F}_{s, t}^{\varepsilon}\left(E_{n}\right)+c_{0} \varepsilon_{0}^{\frac{t}{t-s}}\left|E_{n}\right|\right] \\
& \leqslant 2\left[\mathcal{F}_{s, t}^{\varepsilon}\left(E_{n}\right)+c_{0} \varepsilon_{0}^{\frac{t}{t-s}}|| E_{n}\left|-\omega_{N}\right|+\omega_{N} c_{0} \varepsilon_{0}^{\frac{t}{t-s}}\right] \\
& \leqslant 2\left[\mathcal{G}_{n}\left(E_{n}\right)+\omega_{N} c_{0} \varepsilon_{0}^{\frac{t}{t-s}}\right] \\
& \leqslant 2\left[\mathcal{F}_{s, t}^{\varepsilon}\left(F_{\varepsilon}\right)+\omega_{N} c_{0} \varepsilon_{0}^{\frac{t}{t-s}}\right] \\
& \leqslant 2\left[\mathcal{F}_{s, t}^{\varepsilon}(B)+\omega_{N} c_{0} \varepsilon_{0}^{\frac{t}{t-s}}\right] \\
& \leqslant 2\left[(1-t) P_{t}(B)+\omega_{N} c_{0} \varepsilon_{0}^{\frac{t}{t-s}}\right]
\end{aligned}
$$

recalling that $B$ denotes the ball centered in 0 and radius 1 , with $|B(0,1)|=\omega_{N}$. This gives the bound for $(1-t) P_{t}\left(E_{n}\right)$, and then the bound on $s P_{s}\left(E_{n}\right)$ follows from $(2.3)$. This proves (5.7).

From (5.5) and (5.7) it follows that $\Lambda_{n} \sigma_{n}$ is also uniformly bounded, that is,

$$
\sigma_{n} \leqslant \frac{c_{0}}{\Lambda_{n}} \rightarrow 0 \quad \text { as } n \rightarrow+\infty .
$$

Moreover, for $\sigma_{n} \leqslant 1 / 2$ we have, supposing $\sigma_{n}=\left|E_{n}\right|-\omega_{N}>0$ (the other case can be treated in a similar way),

$$
\left(\frac{\left|E_{n}\right|}{\omega_{N}}\right)^{-\frac{N-s}{N}} \geqslant\left(1+\frac{\sigma_{n}}{\omega_{N}}\right)^{-\frac{N-s}{N}} \geqslant 1-\frac{N-s}{N} \frac{\sigma_{n}}{\omega_{N}}
$$


and similarly

$$
\left(\frac{\left|E_{n}\right|}{\omega_{N}}\right)^{-\frac{N-t}{N}} \leqslant\left(1-\frac{\sigma_{n}}{\omega_{N}}\right)^{-\frac{N-t}{N}} \leqslant 1+C \frac{N-t}{N} \frac{\sigma_{n}}{\omega_{N}}
$$

with $C=C(N, s, t)$. We now define

$$
\tilde{E}_{n}=\left(\frac{\left|E_{n}\right|}{\omega_{N}}\right)^{-\frac{1}{N}} E_{n}
$$

and we use (5.7), (5.8) and $(5.9)$ to obtain

$$
s P_{s}\left(\tilde{E}_{n}\right)=\left(\frac{\left|E_{n}\right|}{\omega_{N}}\right)^{-\frac{N-s}{N}} s P_{s}\left(E_{n}\right) \geqslant\left(1-\frac{N-s}{N} \frac{\sigma_{n}}{\omega_{N}}\right) s P_{s}\left(E_{n}\right) \geqslant s P_{s}\left(E_{n}\right)-c_{0} \sigma_{n},
$$

and

$$
(1-t) P_{t}\left(\tilde{E}_{n}\right)=\left(\frac{\left|E_{n}\right|}{\omega_{N}}\right)^{-\frac{N-t}{N}}(1-t) P_{t}\left(E_{n}\right) \leqslant(1-t) P_{t}\left(E_{n}\right)+c_{0} \sigma_{n}
$$

where the constant $c_{0}$ may differ from line to line.

Therefore, since $\left|\tilde{E}_{n}\right|=\omega_{N}$, the minimality of $F_{\varepsilon}$ gives

$$
\begin{aligned}
\mathcal{F}_{s, t}^{\varepsilon}\left(F_{\varepsilon}\right) & \leqslant \mathcal{F}_{s, t}^{\varepsilon}\left(\tilde{E}_{n}\right)=(1-t) P_{t}\left(\tilde{E}_{n}\right)-\varepsilon s P_{s}\left(\tilde{E}_{n}\right) \\
& \leqslant(1-t) P_{t}\left(E_{n}\right)-\varepsilon s P_{s}\left(E_{n}\right)+c_{0}\left(1+\varepsilon_{0}\right) \sigma_{n} \\
& =\mathcal{F}_{s, t}^{\varepsilon}\left(E_{n}\right)+c_{0}\left(1+\varepsilon_{0}\right) \sigma_{n} .
\end{aligned}
$$

By plugging this into (5.5) we find that

$$
\begin{aligned}
\mathcal{G}_{\varepsilon}\left(E_{n}\right) & <\mathcal{F}_{s, t}^{\varepsilon}\left(F_{\varepsilon}\right) \leqslant \mathcal{F}_{s, t}^{\varepsilon}\left(E_{n}\right)+c_{0}\left(1+\varepsilon_{0}\right) \sigma_{n} \\
& =\mathcal{G}_{\varepsilon}\left(E_{n}\right)-\Lambda_{n} \sigma_{n}+c_{0}\left(1+\varepsilon_{0}\right) \sigma_{n} .
\end{aligned}
$$

We simplify the term $\mathcal{G}_{\varepsilon}\left(E_{n}\right)$ and we divide by $\sigma_{n}$, which is possible thanks to (5.6), we conclude that

$$
0<-\Lambda_{n}+c_{0}\left(1+\varepsilon_{0}\right)
$$

This gives a contradiction for $\Lambda_{n}$ large enough, and proves that $F_{\varepsilon}$ is a minimizer for problem (5.3).

Lemma 5.3. Let $F_{\varepsilon}$ be a minimizer of problem (5.3) with $\varepsilon<\varepsilon_{0}$ and $\Lambda \geqslant \Lambda_{0}$, $\varepsilon_{0}$ and $\Lambda_{0}$ as in Lemma 5.2. and let $E_{\varepsilon}$ be a set of finite perimeter with ||$E_{\varepsilon}\left|-\omega_{N}\right|<1 / \Lambda$. Then,

$$
\begin{aligned}
(1-t) P_{t}\left(F_{\varepsilon}\right) \leqslant & (1-t) P_{t}\left(E_{\varepsilon}\right)+\varepsilon c_{N}\left(1-\frac{s}{t}\right)^{-1}\left|F_{\varepsilon} \Delta E_{\varepsilon}\right|^{1-\frac{s}{t}}\left[(1-t) P_{t}\left(F_{\varepsilon} \Delta E_{\varepsilon}\right)\right]^{\frac{s}{t}} \\
& +\Lambda|| E_{\varepsilon}\left|-\omega_{N}\right| \mid .
\end{aligned}
$$

Proof. Notice that, denoting by $\int_{U}=\int_{U} f$ for a non-negative function $f$, the following computation holds

$$
\int_{F_{\varepsilon}} \int_{F_{\varepsilon}^{c}}=\int_{F_{\varepsilon} \backslash E_{\varepsilon}} \int_{\left(F_{\varepsilon} \cup E_{\varepsilon}\right)^{c}}+\int_{F_{\varepsilon} \backslash E_{\varepsilon}} \int_{E_{\varepsilon} \backslash F_{\varepsilon}}+\int_{F_{\varepsilon} \cap E_{\varepsilon}} \int_{\left(F_{\varepsilon} \cup E_{\varepsilon}\right)^{c}}+\int_{F_{\varepsilon} \cap E_{\varepsilon}} \int_{E_{\varepsilon} \backslash F_{\varepsilon}} .
$$

By interchanging the roles of $F_{\varepsilon}$ and $E_{\varepsilon}$, and setting $f(x, y)=|x-y|^{-(s+N)}$ we get

$$
\begin{aligned}
P_{S}\left(F_{\varepsilon}\right)-P_{s}\left(E_{\varepsilon}\right) & =\int_{F_{\varepsilon} \backslash E_{\varepsilon}} \int_{\left(F_{\varepsilon} \cup E_{\varepsilon}\right)^{c}}-\int_{E_{\varepsilon} \backslash F_{\varepsilon}} \int_{\left(F_{\varepsilon} \cup E_{\varepsilon}\right)^{c}}+\int_{F_{\varepsilon} \cap E_{\varepsilon}} \int_{E_{\varepsilon} \backslash F_{\varepsilon}}-\int_{E_{\varepsilon} \cap F_{\varepsilon}} \int_{F_{\varepsilon} \backslash E_{\varepsilon}} \\
& \leqslant \int_{F_{\varepsilon} \backslash E_{\varepsilon}} \int_{\left(F_{\varepsilon} \cup E_{\varepsilon}\right)^{c}}+\int_{E_{\varepsilon} \backslash F_{\varepsilon}} \int_{F_{\varepsilon} \cap E_{\varepsilon}} \leqslant P_{s}\left(F_{\varepsilon} \Delta E_{\varepsilon}\right) .
\end{aligned}
$$


Therefore, by the minimality of $F_{\varepsilon}$ we get

$$
\begin{aligned}
(1-t) P_{t}\left(F_{\varepsilon}\right) & \leqslant(1-t) P_{t}\left(E_{\varepsilon}\right)+\varepsilon\left[s P_{s}\left(F_{\varepsilon}\right)-s P_{s}\left(E_{\varepsilon}\right)\right]+\Lambda\left(|| E_{\varepsilon}\left|-\omega_{N}\right|-|| F_{\varepsilon}\left|-\omega_{N}\right|\right) \\
& \leqslant(1-t) P_{t}\left(E_{\varepsilon}\right)+\varepsilon s P_{s}\left(F_{\varepsilon} \Delta E_{\varepsilon}\right)+\Lambda|| E_{\varepsilon}\left|-\omega_{N}\right| .
\end{aligned}
$$

Hence the desired result follows from (2.3).

We point out that from Lemma 5.3 it follows that $F_{\varepsilon}$ is a multiplicative $\omega$-minimizer for the $t$-perimeter. In the sequel, as customary, the fractional perimeter of a set $E$ in a ball $B(x, R)$ will be denoted by $P_{t}(E, B(x, R))$.

Corollary 5.4. Let $\varepsilon_{0}$ and $\Lambda_{0}$ be as in Lemma 5.2. Let $F_{\varepsilon}$ be a minimizer of (5.1) with $\varepsilon<\varepsilon_{0}$, let $x \in \partial^{m} F_{\varepsilon}$, and let $E_{\varepsilon}$ be a set of finite t-perimeter with

$$
F_{\varepsilon} \Delta E_{\varepsilon} \subset B(x, R) .
$$

There holds

$$
P_{t}\left(F_{\varepsilon}, B(x, R)\right) \leqslant \frac{1+c_{0} R^{t-s}}{1-c_{0} R^{t-s}} P_{t}\left(E_{\varepsilon}, B(x, R)\right)
$$

for some $c_{0}>0$ and for any $R<R_{0}=R_{0}\left(N, \delta_{0}\right)$.

Proof. We observe that, by direct calculations, from 5.12 , follows

$$
\begin{aligned}
& P_{t}\left(F_{\varepsilon}\right)-P_{t}\left(E_{\varepsilon}\right) & =P_{t}\left(F_{\varepsilon}, B(x, R)\right)-P_{t}\left(E_{\varepsilon}, B(x, R)\right) \\
& \text { and } \quad P_{t}\left(F_{\varepsilon} \Delta E_{\varepsilon}\right) & \leqslant P_{t}\left(F_{\varepsilon}, B(x, R)\right)+P_{t}\left(E_{\varepsilon}, B(x, R)\right) .
\end{aligned}
$$

Furthermore, thanks to Lemma 5.2 we know that $F_{\varepsilon}$ is also a minimizer of $(5.3)$, with $\Lambda=\Lambda_{0}$. From (5.10) and the fractional isoperimetric inequality (3.7), we then get

$$
\begin{aligned}
(1-t) P_{t}\left(F_{\varepsilon}, B(x, R)\right) \leqslant & (1-t) P_{t}\left(E_{\varepsilon}, B(x, R)\right) \\
& +\varepsilon_{0} c_{N}\left(1-\frac{s}{t}\right)^{-1}\left[c_{N} t\right]^{1-\frac{s}{t}}\left|F_{\varepsilon} \Delta E_{\varepsilon}\right|^{\frac{t-s}{N}}(1-t) P_{t}\left(F_{\varepsilon} \Delta E_{\varepsilon}\right) \\
& +\Lambda_{0}|| E_{\varepsilon}\left|-\omega_{N}\right| .
\end{aligned}
$$

Moreover, again from the fractional isoperimetric inequality and using (5.12),

$$
\begin{aligned}
\Lambda_{0}|| E_{\varepsilon}\left|-\omega_{N}\right| & =\Lambda_{0}||\left|E_{\varepsilon}\right|-\omega_{N}|-|\left|F_{\varepsilon}\right|-\omega_{N}|| \\
& \leqslant \Lambda_{0}\left|F_{\varepsilon} \Delta E_{\varepsilon}\right|^{\frac{N-t}{N}}\left|F_{\varepsilon} \Delta E_{\varepsilon}\right|^{\frac{t}{N}} \\
& \leqslant c_{N} \Lambda_{0} t(1-t) P_{t}\left(F_{\varepsilon} \Delta E_{\varepsilon}\right)\left|F_{\varepsilon} \Delta E_{\varepsilon}\right|^{\frac{t}{N}} \\
& \leqslant c_{N} \Lambda_{0} t(1-t) P_{t}\left(F_{\varepsilon} \Delta E_{\varepsilon}\right) R^{t} .
\end{aligned}
$$

From this, 5.14 and 5.15 we arrive at

$$
\begin{aligned}
(1-t) P_{t}\left(F_{\varepsilon}, B(x, R)\right) \leqslant & (1-t) P_{t}\left(E_{\varepsilon}, B(x, R)\right) \\
& +\varepsilon_{0} c_{N}\left(1-\frac{s}{t}\right)^{-1}\left[c_{N} t\right]^{1-\frac{s}{t}} R^{t-s}(1-t) P_{t}\left(F_{\varepsilon} \Delta E_{\varepsilon}\right) \\
& +\Lambda_{0} c_{N} t R^{t}(1-t) P_{t}\left(F_{\varepsilon} \Delta E_{\varepsilon}\right) \\
\leqslant & (1-t) P_{t}\left(E_{\varepsilon}, B(x, R)\right) \\
& +c_{0} R^{t-s}(1-t)\left[P_{t}\left(F_{\varepsilon}, B(x, R)\right)+P_{t}\left(E_{\varepsilon}, B(x, R)\right)\right]
\end{aligned}
$$


which gives (5.13), if $R<\min \left\{1,1 / c_{0}^{\frac{1}{t-s}}\right\}=: R_{0}$, with

$$
c_{0}:=\varepsilon_{0} c_{N}\left(1-\frac{s}{t}\right)^{-1}\left[c_{N} t\right]^{1-\frac{s}{t}}+\Lambda_{0} c_{N} t
$$

Lemma 5.5. There exists $\Theta=\Theta\left(N, \delta_{0}\right)>0$ and $R_{0}=R_{0}\left(N, \delta_{0}\right)>0$ such that, for any $x \in \partial^{m} F_{\varepsilon}$ and $R<R_{0}$, there holds

$$
(1-t) P_{t}\left(F_{\varepsilon}, B(x, R)\right) \leqslant \Theta R^{N-t} .
$$

Proof. Let $E_{\varepsilon}=F_{\varepsilon} \backslash B(x, R)$, and observe that $P_{t}\left(E_{\varepsilon}, B(x, R)\right) \leqslant P_{t}(B(x, R))$. From (5.13), possibly reducing $R_{0}$, we then get

$$
(1-t) P_{t}\left(F_{\varepsilon}, B(x, R)\right) \leqslant\left(1+c_{0} R^{t-s}\right)(1-t) P_{t}(B(x, R)) \leqslant \Theta R^{N-t} .
$$

From Lemma 5.5 it follows that $F_{\varepsilon}$ is also an additive $\omega$-minimizer for the $t$-perimeter.

Corollary 5.6. Let $\varepsilon_{0}$ be as in Lemma 5.2. Let $F_{\varepsilon}$ be a minimizer of (5.1) with $\varepsilon<\varepsilon_{0}$, let $x \in \partial^{m} F_{\varepsilon}$, and let $E_{\varepsilon}$ be a set of finite t-perimeter with

$$
F_{\varepsilon} \Delta E_{\varepsilon} \subset B(x, R) \text {. }
$$

There holds

$$
(1-t) P_{t}\left(F_{\varepsilon}, B(x, R)\right) \leqslant(1-t) P_{t}\left(E_{\varepsilon}, B(x, R)\right)+c_{0} R^{N-s}
$$

for any $R<R_{0}$, with $R_{0}, c_{0}$ depending only on $N, \delta_{0}$.

Proof. By (5.13) and (5.16), possibly increasing the constant $c_{0}$ we have

$(1-t) P_{t}\left(E_{\varepsilon}, B(x, R)\right) \geqslant\left(1-c_{0} R^{t-s}\right)(1-t) P_{t}\left(F_{\varepsilon}, B(x, R)\right) \geqslant(1-t) P_{t}\left(F_{\varepsilon}, B(x, R)\right)-c_{0} \Theta R^{N-s}$

for any $R<R_{0}$.

From Corollary 5.6 we derive the $C^{1, \beta}$ regularity minimizer of (5.1) following standard arguments that can be found in [12, Theorem 1] (see also [18, Corollary 3.5]).

Corollary 5.7. There exists $\beta=\beta\left(N, \delta_{0}\right)<1$ such that any minimizer $F_{\varepsilon}$ of (5.1), with $\varepsilon<\varepsilon_{0}$, as in Lemma 5.2. has boundary of class $C^{1, \beta}$ outside of a closed singular set of Hausdorff dimension at most $N-2$.

Remark 5.8. If $t=1$, by the general regularity theory for $\omega$-minimizers of the classical perimeter developed in [4, 35] we have that $F_{\varepsilon}$ has boundary of class $C^{1, \beta}$ outside of a closed singular set of Hausdorff dimension at most $N-8$.

We are in the position of completing the proof of Theorem 1.2 .

Proof of Theorem 1.2. The existence follows from Theorem 4.2. The regularity of $\partial F$ follows from Corollary 5.4 and Corollary 5.7. 


\section{RIGIDITY OF MINIMIZERS FOR SMALL VOLUMES AND PROOF OF THE MAIN THEOREM}

We now develop the rigidity theory needed to prove Theorems 1.3 and 1.1 .

Theorem 6.1. For any $\eta>0$ there exists $\bar{\varepsilon}=\bar{\varepsilon}\left(\eta, N, \delta_{0}\right)>0$ such that any minimizer $F_{\varepsilon}$ of (5.1), with $\varepsilon<\bar{\varepsilon}$, can be written as

$$
\partial F_{\varepsilon}=\left\{\left(1+u_{\varepsilon}(x)\right) x: x \in \partial B\right\},
$$

where $B$ is the ball of radius 1 having the same barycenter of $F_{\varepsilon}$, and $u_{\varepsilon}: \partial B \rightarrow \mathbb{R}$ satisfies

$$
\left\|u_{\varepsilon}\right\|_{C^{1}(\partial B)} \leqslant \eta \text {. }
$$

Proof. From Lemma 3.13 , putting $m=\varepsilon^{\frac{N}{t-s}} \omega_{N}$ there, it follows that $\left|F_{\varepsilon} \Delta B\right| \rightarrow 0$ as $\varepsilon \rightarrow 0$. From the density lower bound proved in Proposition 3.11 it then follows that $\partial F_{\varepsilon} \rightarrow \partial B$ in the Hausdorff topology. The result now follows via a standard argument based on the $\omega$-minimality of $F_{\varepsilon}$ and on the regularity of the limit set $B$ (see [18, Corollary 3.6] and, for $t=1$, [35, Theorem 1] and [30, Theorem 26.6]).

Theorem 6.2. There exist $\tau_{0}, c_{1}, c_{2}>0$ depending only on $N$, with $c_{1}<c_{2}$, with the following property. Suppose that $E_{\tau}$ is such that, for $\tau \in\left[0, \tau_{0}\right], \partial E_{\tau}$ takes the form

$$
\partial E_{\tau}=\{(1+\tau u(x)) x: x \in \partial B\}
$$

where $u: \partial B \rightarrow \mathbb{R}$ satisfies

$$
\|u\|_{C^{1}(\partial B)} \leqslant 1 / 2 \text {. }
$$

Suppose moreover that the barycenter of $E_{\tau}$ is the same of that of $B$, say 0 , and that $\left|E_{\tau}\right|=|B|$. Then, for all $\alpha \in(0,1)$ it holds true that

$$
c_{1} \tau^{2}\left([u]_{H^{\frac{1+\alpha}{2}}(\partial B)}^{2}+\alpha P_{\alpha}(B)\|u\|_{L^{2}(\partial B)}^{2}\right) \leqslant P_{\alpha}\left(E_{\tau}\right)-P_{\alpha}(B) \leqslant c_{2} \tau^{2}[u]_{H^{\frac{1+\alpha}{2}}}^{2}(\partial B) .
$$

Proof. The first inequality in (6.2) has been proved in [18, Theorem 2.1]. It remains to prove the second inequality.

As in [18, Formula (2.20)], after some calculations we get that

$$
P_{\alpha}\left(E_{\tau}\right)=\frac{\tau^{2}}{2} g(\tau)+\frac{P_{\alpha}(B)}{P(B)} h(\tau),
$$

where we set

$$
h(\tau):=\int_{\partial B}(1+\tau u(x))^{N-\alpha} d \mathcal{H}^{N-1}(x),
$$

and

$$
g(\tau):=\int_{\partial B} \int_{\partial B}\left(\int_{u(y)}^{u(x)} \int_{u(y)}^{u(x)} f_{|x-y|}(1+\tau r, 1+\tau \rho) d r d \rho\right) d \mathcal{H}^{N-1}(x) d \mathcal{H}^{N-1}(y)
$$

being

$$
f_{\theta}(a, b):=\frac{a^{N-1} b^{N-1}}{\left(|a-b|^{2}+a b \theta^{2}\right)^{\frac{N+\alpha}{2}}} .
$$

We observe that $r$ and $\rho$ in the definition of $g$ range in $\left[-\|u\|_{L^{\infty}(\partial B)},\|u\|_{L^{\infty}(\partial B)}\right] \subseteq[-1,1]$, since $\|u\|_{L^{\infty}(\partial B)} \leqslant 1$. Hence, comparing with the definition of $g$, we notice that $a$ and $b$ in (6.4) range in $[1-\tau, 1+\tau]$, and therefore they are bounded and bounded away from zero. As a consequence, we get

$$
f_{\theta}(a, b) \leqslant \frac{C_{1}}{\left(C_{2}+C_{3} \theta^{2}\right)^{\frac{N+\alpha}{2}}} \leqslant \frac{C_{1}}{\left(C_{3} \theta^{2}\right)^{\frac{N+\alpha}{2}}}=\frac{C_{4}}{\theta^{N+\alpha}}
$$


for suitable constants $C_{1}, \ldots, C_{4}>0$. Therefore, up to renaming the constants, we have

$$
g(\tau) \leqslant \int_{\partial B} \int_{\partial B}\left(\int_{u(y)}^{u(x)} \int_{u(y)}^{u(x)} \frac{c_{N}}{|x-y|^{N+\alpha}} d r d \rho\right) d \mathcal{H}^{N-1}(x) d \mathcal{H}^{N-1}(y)=c_{N}[u]_{H^{\frac{1+\alpha}{2}}(\partial B)}^{2} .
$$

Thus, since $h(0)=P(B)$, by 6.3 we get

$$
P_{\alpha}\left(E_{\tau}\right)-P_{\alpha}(B) \leqslant c_{N} \tau^{2}[u]_{H^{\frac{1+\alpha}{2}}(\partial B)}^{2}+\frac{P_{\alpha}(B)}{P(B)}(h(\tau)-h(0)) .
$$

Now we want to estimate $h(\tau)-h(0)$. Since $\left|E_{\tau}\right|=|B|$, using polar coordinates, we get

$$
\int_{\partial B}(1+\tau u)^{N} d \mathcal{H}^{N-1}=N\left|E_{\tau}\right|=N|B|=P(B) .
$$

Thus

$$
h(\tau)-h(0)=\int_{\partial B}(1+\tau u)^{N-\alpha} d \mathcal{H}^{N-1}-P(B)=\int_{\partial B}(1+\tau u)^{N}\left((1+\tau u)^{-\alpha}-1\right) d \mathcal{H}^{N-1}
$$

By a Taylor expansion, we know that for any $x \geqslant 0$ small enough, it holds

$$
\begin{aligned}
& \left.\left((1+x)^{-\alpha}-1\right)(1+x)^{N}\right) \\
& \quad=\left(-\alpha x+\frac{\alpha(\alpha+1)}{2} x^{2}+\alpha \beta(x)\right)\left(1+N x+\frac{N(N-1)}{2} x^{2}+\gamma(x)\right),
\end{aligned}
$$

with $|\beta(x)|+|\gamma(x)| \leqslant c_{N} x^{3}$, so that

$$
\left.\left((1+x)^{-\alpha}-1\right)(1+x)^{N}\right) \leqslant-\alpha x+\left(\frac{\alpha(\alpha+1)}{2}-N \alpha\right) x^{2}+\alpha c_{N} x^{3} .
$$

By applying such an inequality to 6.7 , and using the fact that $\|u\|_{L^{\infty}(\partial B)}<1$, we get

$$
h(\tau)-h(0) \leqslant-\alpha \int_{\partial B}\left[\tau u+\left(N-\frac{\alpha+1}{2}\right) \tau^{2} u^{2}\right] d \mathcal{H}^{N-1}+\alpha c_{N} \tau^{3}\|u\|_{L^{2}(\partial B)}^{2}
$$

Also, from (6.6), we have

$$
0=\int_{\partial B}\left((1+\tau u)^{N}-1\right) d \mathcal{H}^{N-1} \leqslant \int_{\partial B}\left(N \tau u+N(N-1) \tau^{2} u^{2}+c_{N} \tau^{3} u^{3}\right) d \mathcal{H}^{N-1} .
$$

Hence, since $\|u\|_{L^{\infty}(\partial B)}<1$, we obtain

$$
-\int_{\partial B} \tau u d \mathcal{H}^{N-1} \leqslant \frac{N-1}{2} \tau^{2}\|u\|_{L^{2}(\partial B)}^{2}+c_{N} \tau^{3}\|u\|_{L^{2}(\partial B)}^{2},
$$

so that 6.8 gives

$$
h(\tau)-h(0) \leqslant-\frac{\tau^{2}}{2} \alpha(N-\alpha)\|u\|_{L^{2}(\partial B)}^{2}+\alpha c_{N} \tau^{3}\|u\|_{L^{2}(\partial B)}^{2} \leqslant 0
$$

for $\tau \leqslant \tau_{0}(N)$. By inserting this into 6.5 we obtain the second inequality in 6.2 .

We now complete the proof of Theorem 1.3 .

Proof of Theorem 1.3. We have to show that there exists $\varepsilon_{1}=\varepsilon_{1}\left(N, \delta_{0}\right) \in\left(0, \varepsilon_{0}\right], \varepsilon_{0}$ as in (5.4), and so $\bar{m}_{1}=\bar{m}_{1}\left(N, \delta_{0}\right) \in\left(0, \bar{m}_{0}\right]$, such that the ball $B$ is the only minimizer of problem (5.1) for $\varepsilon<\varepsilon_{1}$. Let $\varepsilon<\varepsilon_{1}$ and let $F_{\varepsilon}$ be a minimum of problem (5.1), which exists by Theorem 1.2 . By the minimality of $F_{\varepsilon}$ we have

$$
(1-t) P_{t}\left(F_{\varepsilon}\right)-(1-t) P_{t}(B) \leqslant \varepsilon\left(s P_{s}\left(F_{\varepsilon}\right)-s P_{s}(B)\right)
$$


where $B$ has the same barycenter of $F_{\varepsilon}$. Possibly reducing $\varepsilon$ we can assume that $\partial F_{\varepsilon}$ can be written as in (6.1), with $\left\|u_{\varepsilon}\right\|_{C^{1}(\partial B)} \leqslant \tau_{0} / 2$, where $\tau_{0}$ is as in Theorem 6.2. Then, from (6.9) and 6.2 it follows

$$
\begin{aligned}
c_{1}(1-t)\left[u_{\varepsilon}\right]_{H^{\frac{1+t}{2}}(\partial B)}^{2} & \leqslant c_{1}(1-t)\left(\left[u_{\varepsilon}\right]_{H^{\frac{1+t}{2}}(\partial B)}^{2}+t P_{t}(B)\left\|u_{\varepsilon}\right\|_{L^{2}(\partial B)}^{2}\right) \\
& \leqslant\left((1-t) P_{t}\left(F_{\varepsilon}\right)-(1-t) P_{t}(B)\right) \\
& \leqslant \varepsilon\left(s P_{s}\left(F_{\varepsilon}\right)-s P_{s}(B)\right) \\
& \leqslant \varepsilon s c_{2}\left[u_{\varepsilon}\right]_{H^{\frac{1+s}{2}}}^{2}(\partial B)
\end{aligned}
$$

From 2.17$)$ it then follows

$$
c_{1}(1-t)\left[u_{\varepsilon}\right]_{H^{\frac{1+t}{2}}(\partial B)}^{2} \leqslant c_{N} \frac{\varepsilon s}{(1-s)}(1-t)\left[u_{\varepsilon}\right]_{H^{\frac{1+t}{2}}(\partial B)}^{2}
$$

which implies $u_{\varepsilon}=0$, that is $F_{\varepsilon}=B$, whenever $\varepsilon$ is sufficiently small.

The next result is the counterpart to Theorem 1.3 for large volumes.

Theorem 6.3. For all $0<s<t \leqslant 1$, there exists a volume $\bar{m}_{2}=\bar{m}_{2}(N, s, t) \geqslant \bar{m}_{1}$ such that, for $m>m_{2}$, the ball is not a local minimizer of problem (1.7).

Proof. We have to show that there exists $\varepsilon_{2} \geqslant \varepsilon_{1}$ such that the ball $B$ is not a local minimizer of problem 5.1 for $\varepsilon>\varepsilon_{2}$. We look for a competitor $F_{\varepsilon} \neq B$ which can be written as in 6.1), with $u \neq \equiv 0$ and and $\|u\|_{C^{1}(\partial B)} \leqslant \tau_{0} / 2$, where $\tau_{0}$ is as in Theorem 6.2. As above, from (6.2) it follows

$$
\begin{aligned}
\left((1-t) P_{t}\left(F_{\varepsilon}\right)-(1-t) P_{t}(B)\right) & \leqslant c_{2}(1-t)[u]_{H^{\frac{1+t}{2}}}^{2}(\partial B) \\
& <\varepsilon c_{1} s[u]_{H^{\frac{1+s}{2}}}^{2}(\partial B) \\
& \leqslant \varepsilon\left(s P_{s}\left(F_{\varepsilon}\right)-s P_{s}(B)\right)
\end{aligned}
$$

as soon as

$$
\varepsilon>\varepsilon_{2}:=\frac{c_{2}(1-t)[u]_{H^{\frac{1+t}{2}}(\partial B)}^{2}}{c_{1} s[u]_{H^{\frac{1+s}{2}}}^{2}(\partial B)} .
$$

This shows that $F_{\varepsilon}$ has lower energy than $B$, so that the ball cannot be a local minimizer of problem (1.7).

Notice that $\lim _{s \rightarrow 0} \bar{m}_{2}(N, s, t)=+\infty$ for all $t \in(0,1]$, which is consistent with the fact that the ball is the unique minimizer of the $t$-perimeter, with volume constraint.

We conclude the section with the proof of Theorem 1.1 .

Proof of Theorem 1.1. Let $E \subset \mathbb{R}^{N}$. Since the deficit of a set is a 0 homogeneous quantity, we can suppose that the set $E$ has measure $\omega_{N}$. Because of this, inequality 1.6 is equivalent to prove that there exists $C(N, s, t)>0$ such that the inequality

$$
\widetilde{\delta} P_{t}(E):=P_{t}(E)-P_{t}(B) \geqslant C(N, s, t)\left(P_{s}(E)-P_{s}(B)\right) .
$$


holds true for any set $E$ of measure $\omega_{N}$. Indeed if this is true, then we get that for any $E \subset \mathbb{R}^{N}$ it holds

$$
\begin{aligned}
\delta P_{t}(E) & =\delta P_{t}\left(E \omega_{N} /|E|\right)=\frac{P_{t}\left(E \omega_{N} /|E|\right)-P_{t}(B)}{P_{t}(B)} \\
& \geqslant C(N, s, t) \frac{P_{s}\left(E \omega_{N} /|E|\right)-P_{s}(B)}{P_{t}(B)}=C(N, s, t) \frac{P_{s}(B)}{P_{t}(B)} \delta P_{s}(E) .
\end{aligned}
$$

that is exactly 1.6 with $C=C(N, s, t) \frac{P_{s}(B)}{P_{t}(B)}$.

Let $E \subset \mathbb{R}^{N}$ be a set of measure $\omega_{N}$. By Theorem 1.2 we know that there exists $\varepsilon_{0}=\varepsilon_{0}(N, s, t)$ such that if $\varepsilon \leqslant \varepsilon_{0}$ then the only minimizer of the problem

$$
\min \left\{(1-t) P_{t}(E)-\varepsilon s P_{s}(E):|E|=\omega_{N}\right\}
$$

is given by the unit ball $B$. This entails that

$$
\widetilde{\delta} P_{t}(E) \geqslant \frac{\varepsilon_{0} s}{1-t} \widetilde{\delta} P_{s}(E) .
$$

\section{A fRACtional isoperimetric PROBlem}

We recall from the Introduction the definition of the functional $\widetilde{\mathcal{F}_{s, t}}$ given by

$$
\widetilde{\mathcal{F}_{s, t}}(E)= \begin{cases}\frac{\left((1-t) P_{t}(E)\right)^{N-s}}{\left(s P_{s}(E)\right)^{N-t}} & \text { if } 0<s<t<1 \\ \frac{\left(N \omega_{N} P(E)\right)^{N-s}}{\left(s P_{s}(E)\right)^{N-1}} & \text { if } 0<s<t=1 \\ \frac{(1-t) P_{t}(E)^{N}}{\left(N \omega_{N}|E|\right)^{N-t}} & \text { if } 0=s<t<1 \\ N \omega_{N} \frac{P(E)^{N}}{|E|^{N-1}} & \text { if } s=0 \text { and } t=1 .\end{cases}
$$

In this section we consider the generalized isoperimetric problem

$$
\min _{E \subset \mathbb{R}^{N}} \widetilde{\mathcal{F}_{s, t}}(E), \quad 0 \leqslant s<t \leqslant 1 .
$$

Remark 7.1. Notice that the quantity in 7.1 is scale invariant, hence without loss of generality we can look for minimizers $E$ satisfying a volume constraint $|E|=\omega_{N}$.

The main aim of this section is the following existence theorem.

Theorem 7.2. There exists a minimizer of problem (7.1).

Since its proof may result technical, for the reader's convenience we begin with a description of its strategy.

\section{Strategy of the proof}

An usually successful argument to get existence for isoperimetric-type problems is the following: first apply the Direct Method in the Calculus of Variations on minimizing sequences which are equibounded, that is, whose elements are contained in a prescribed ball of fixed radius. Then try to show that starting from a given minimizing sequence $E_{n}$ it is possible to construct another minimizing sequence $F_{n}$ which is uniformly bounded and make use of the first step to conclude. 
Unfortunately it seems that the second step is not easily applicable to the functional $\widetilde{\mathcal{F}_{s, t}}$, for $s>0$. An hint about what kind of difficulties may occur is the following: it is not even clear if a minimizer (if any) is connected or not. Indeed, up to the case $s=0$, where $s P_{s}$ reduces to the Lebesgue measure, which is translation invariant, the denominator of $\widetilde{\mathcal{F}_{s, t}}$ acts as a disaggregating term among different connected components. On the other hand it is not evident when the numerator (which tends to aggregate different connected components) can overcome such an effect. For this reason we adopt a different strategy from that described above, which can be divided as well into two steps.

Step 1 The first step is very easy and it is similar to that mentioned above. It reduces to show that for each given $R>0$ there exists a minimizer $E_{R}$ for $\widetilde{\mathcal{F}_{s, t}}$ among sets contained in a cube $Q_{R}=[-R, R]^{N}$ and that $P_{t}\left(E_{R}\right)$ is bounded from above independently from $R$. This is done in done in Lemmas 7.4 and 7.5 .

Step 2 The second step, developed in the very proof of Theorem 7.2, is longer and needs a more careful analysis. Since, as mentioned above, we are not able to show that a minimizing sequence can be uniformly bounded, we adopt a different idea that may be seen as an adaptation of a concentration-compactness technique à la Lions, where the compactness is replaced by a sort of selection principle. More precisely we consider, for $n \in \mathbb{N}$, the minimizer $E_{n} \subseteq[-n, n]^{N}$ found in Step 1 and require them to have prescribed mass, say 1. Since we do not know, as $n$ increases, the behavior of the $E_{n}$ 's (e.g. if they are connected, equibounded...) we select, for every $n$ all the unitary cubes in $\mathbb{R}^{N}$ of the form $[-1,1]^{N}+z, z \in \mathbb{Z}^{N}$, which have a non-negligible intersection with $E_{n}$. So for each $n$ we get a set of cubes $Q_{i, 1} \ldots, Q_{i, k_{n}}$ with non-empty intersection with $E_{n}$. Clearly for two fixed indexes $i \neq j$ we may have that $\operatorname{dist}\left(Q_{i, n}, Q_{j, n}\right)$ diverges to $+\infty$. This means that two components of $E_{n}$ will have infinity distance as $n \rightarrow \infty$ (this phenomenon may be seen as a dichotomy phenomenon). If this does not happens, then we say that $Q_{i, n}$ adn $Q_{j, n}$ have finite mutual distance at infinity and we (suitably) collect them together. Such a construction, thanks to the equiboundedness of $P_{t}\left(E_{n}\right)$, allows us to construct a sequence of limit points $\left\{G_{i}\right\}_{i \in \mathbb{N}}$ of the $E_{n}$ 's, where $G_{i}$ is just one of the collections of cubes with finite mutual distance at infinity. Now the idea is simple: first we need to show that the amount of measure of all the $G_{i}$ is the same as that of the $E_{n}$ 's (so, we want to eliminate the vanishing phenomenon). Then, we want to select a $G_{i}$ such that $\widetilde{\mathcal{F}_{s, t}}\left(G_{i}\right)$ is the lowest possible. It is not difficult then to conclude that $G_{i}$ is a minimizer for $\widetilde{\mathcal{F}_{s, t}}$.

We begin now the proof with a namely a suitable version of the isoperimetric inequality (Lemma 7.4) and an existence result with uniform estimates for a constrained minimization problem (Lemma 7.5).

Remark 7.3. In what follows, with a slight abuse of notation, we extend the functionals $(1-$ t) $P_{t}(\cdot)$ and $s P_{s}(\cdot)$ to $t=1$ and $s=0$ respectively, meaning that for $t=1$ it equals $N \omega_{N} P(\cdot)$, while for $s=0$ it equals $N \omega_{N}|\cdot|$.

Lemma 7.4. Let $s<t \in[0,1]$ satisfy 3.1]. For any $E \subset \mathbb{R}^{N}$ there holds

$$
\frac{\left((1-t) P_{t}(E)\right)^{\frac{N-s}{N-t}}}{\left(s P_{s}(E)\right)} \geqslant c
$$

for some $c=c\left(N, \delta_{0}\right)>0$. 
Proof. Let $s<t \in[0,1]$ and let $\delta_{0}=t-s$. Notice that

$$
\frac{t}{t-s}=1+\frac{s}{t-s} \leqslant 1+\frac{1}{\delta_{0}}
$$

Then from 2.15), and since $\delta_{0}<t$, it follows

$$
|E|^{1-\frac{s}{t}} \leqslant C\left(N, \delta_{0}\right)\left((1-t) P_{t}(E)\right)^{\frac{N(t-s)}{(N-t) t}} .
$$

Plugging this estimate into $(2.3)$ (or $(2.9)$ if $t=1$ ) we get

$$
s P_{s}(E) \leqslant C\left(N, \delta_{0}\right) \frac{t}{t-s}\left((1-t) P_{t}(E)\right)^{\frac{N-s}{N-t}},
$$

which, together with 7.3 gives 7.2 .

We notice that, if $s=0$, the claim is an immediate consequence of the the fractional isoperimetric inequality 2.15.

Lemma 7.5. Let $s<t \in[0,1]$ satisfy 3.1$]$. For $R>1$ let $Q_{R}=[-R, R]^{N}$. Then, there exists a minimizer $E_{R}$ of the problem

$$
\min _{E \subset Q_{R}|E|=m} \frac{\left((1-t) P_{t}(E)\right)^{N-s}}{\left(s P_{s}(E)\right)^{N-t}} .
$$

Moreover

$$
(1-t) P_{t}\left(E_{R}\right) \leqslant C
$$

where $C$ is independent of $R$.

Proof. We recall that, thanks to the notation introduced in Remark 7.3 we can deal at once with the cases $t<1$ and $t=1$. By Lemma 7.4 we know that

$$
C(R)=\inf _{E \subset Q_{R}|E|=m} \frac{\left((1-t) P_{t}(E)\right)^{\frac{N-s}{N-t}}}{\left(s P_{s}(E)\right)}
$$

is a strictly positive quantity. Clearly the map $R \mapsto C(R)$ is non-increasing. Let $C=C(1)+1$ and let $E_{n}$ be a minimizing sequence for $(7.4)$, so that for $n$ big enough it holds $(1-t) P_{t}\left(E_{n}\right) \leqslant$ $C\left(s P_{s}\left(E_{n}\right)\right)^{(N-t) /(N-s)}$. Possibly increasing the constant $C$, from $(2.3)$ (or $(2.9)$ if $t=1$ ) it follows

$$
(1-t) P_{t}\left(E_{n}\right) \leqslant C\left((1-t) P_{t}\left(E_{n}\right)\right)^{\frac{s(N-t)}{t(N-s)}}
$$

which gives

$$
(1-t) P_{t}\left(E_{n}\right) \leqslant C \quad \text { for all } n .
$$

The existence of a minimizer now follows by the direct method the calculus of variations, since the compact embedding of $L^{1}\left(Q_{R}\right)$ and $H^{s}\left(Q_{R}\right)$ into $H^{t}\left(Q_{R}\right)$ and the estimate 7.5 directly follows from (7.6).

We now prove Theorem 7.2 .

Proof of Theorem 7.2. If $s=0$ then the claim of the theorem is equivalent to that of the isoperimetric inequality (the fractional isoperimetric inequality if $t<1$ ). Thus we consider just the case $s>0$. Again, we shall always write $(t-1) P_{t}$ meaning that such a functional is equivalent to the classical perimeter if $t=1$ (see Remark 7.3). 
Let $E_{n}$ be a minimizer of (7.4) with $R=n \in \mathbb{N}$ and $m=1 / 2$. We divide $Q_{n}$ into $(2 n)^{N}$ unit cubes with vertices in $\mathbb{Z}^{N}$, and we let $\left\{Q_{i, n}\right\}_{i=1}^{I_{n}}$ be the unit cubes with non-negligible intersection with $E_{n}$, that is, $x_{i, n}=\left|E_{n} \cap Q_{i, n}\right| \in(0,1 / 2]$ for all $i \in\left\{1, \ldots, I_{n}\right\}$, for some $I_{n} \in\left\{1, \ldots,(2 n)^{N}\right\}$.

We remark that, from (2.10) (and omitting the integrands for simplicity), we have that

$$
\sum_{i=1}^{\infty} P_{t}\left(E_{n}, Q_{i, n}\right)=\sum_{i=1}^{\infty} \int_{E_{n} \cap Q_{i, n}} \int_{\mathbb{R}^{N} \backslash E_{n}}+\int_{Q_{i, n} \backslash E_{n}} \int_{E_{n} \backslash Q_{i, n}} \leqslant \int_{E_{n} \cap Q_{n}} \int_{\mathbb{R}^{N} \backslash E_{n}}+\int_{Q_{n} \backslash E_{n}} \int_{E_{n}},
$$

which implies that

$$
\sum_{i=1}^{\infty} P_{t}\left(E_{n}, Q_{i, n}\right) \leqslant 2 \int_{E_{n}} \int_{\mathbb{R}^{N} \backslash E_{n}}=2 P_{t}\left(E_{n}\right) .
$$

Now, up to reordering the cubes $Q_{i, n}$ we can assume that the sequence $\left\{x_{i, n}\right\}_{i=1}^{I_{n}}$ is non-increasing in $i$, and we set $x_{i, n}:=0$ for $i>I_{n}$. We have that

$$
\sum_{i=1}^{\infty} x_{i, n}=\frac{1}{2}
$$

and, recalling (2.12), (7.5) and (7.7), and the fact that $x_{i, n} \leqslant\left|E_{n}\right|=1 / 2=\left|Q_{i, n}\right| / 2$, we get

$$
\sum_{i=1}^{\infty} x_{i, n}^{\frac{N-t}{N}} \leqslant C \sum_{i=1}^{\infty}(1-t) P_{t}\left(E_{n}, Q_{i, n}\right) \leqslant 2 C(1-t) P_{t}\left(E_{n}\right) \leqslant C
$$

up to renaming $C$. As in [25, Lemma 4.2], from (7.8) and (7.9) it follows that

$$
\sum_{i=k}^{\infty} x_{i, n} \leqslant C k^{-\frac{1}{N}}
$$

for all $k \in \mathbb{N}$, where $C$ depends only on $(N, s, t)$.

Up to extracting a subsequence (using either a diagonal process or Tychonoff Theorem), we can suppose that $x_{i, n} \rightarrow \alpha_{i} \in[0,1 / 2]$ as $n \rightarrow+\infty$ for every $i \in \mathbb{N}$, so that by $(7.8)$ and (7.10) we have

$$
\sum_{i} \alpha_{i}=\frac{1}{2}
$$

Fix now $z_{i, n} \in Q_{i, n}$. Up to extracting a further subsequence, we can suppose that $d\left(z_{i, n}, z_{j, n}\right) \rightarrow$ $c_{i j} \in[0,+\infty]$, and (recalling (7.5) ) that there exists $G_{i} \subseteq \mathbb{R}^{N}$ such that

$$
\left(E_{n}-z_{i, n}\right) \rightarrow G_{i} \quad \text { in the } L_{\text {loc }}^{1} \text {-convergence }
$$

for every $i \in \mathbb{N}$. We say that $i \sim j$ if $c_{i j}<+\infty$ and we denote by $[i]$ the equivalence class of $i$. Notice that $G_{i}$ equals $G_{j}$ up to a translation, if $i \sim j$. Let $\mathcal{A}:=\{[i]: i \in \mathbb{N}\}$. We claim that

$$
\sum_{[i] \in \mathcal{A}} P_{t}\left(G_{i}\right) \leqslant \liminf _{n \rightarrow+\infty} P_{t}\left(E_{n}\right) \quad \text { and } \quad \sum_{[i] \in \mathcal{A}} P_{s}\left(G_{i}\right) \leqslant \liminf _{n \rightarrow+\infty} P_{s}\left(E_{n}\right) .
$$

To prove it, we first fix $M \in \mathbb{N}$ and $R>0$. We take different equivalent classes $i_{1}, \ldots, i_{M}$ and we notice that if $i_{k} \neq i_{j}$ then the set $z_{i_{k}, n}+Q_{R}$ is drifting far apart from $z_{i_{j}, n}+Q_{R}$, and so

$$
\lim _{n \rightarrow+\infty} \int_{z_{i_{k}, n}+Q_{R}} \int_{z_{i_{j}, n}+Q_{R}} \frac{d x d y}{|x-y|^{N+t}}=0 .
$$


Accordingly, by (2.11), (7.12) and the lower semicontinuity of the perimeter,

$$
\begin{aligned}
\sum_{i=1}^{M} P_{t}\left(G_{i}, Q_{R}\right) & \leqslant \liminf _{n \rightarrow+\infty} \sum_{k=1}^{M} P_{t}\left(E_{n},\left(z_{i_{k}, n}+Q_{R}\right)\right) \\
& \leqslant \liminf _{n \rightarrow+\infty} P_{t}\left(E_{n}, \bigcup_{k=1}^{N}\left(z_{i_{k}, n}+Q_{R}\right)\right)+2 \sum_{\substack{1 \leqslant k, j \leqslant M \\
i_{k} \neq i_{j}}} \int_{z_{i_{k}, n}+Q_{R}} \int_{z_{i_{j}, n}+Q_{R}} \frac{d x d y}{|x-y|^{N+t}} \\
& \leqslant \liminf _{n \rightarrow+\infty} P_{t}\left(E_{n}\right) .
\end{aligned}
$$

By sending first $R \rightarrow+\infty$ and then $M \rightarrow+\infty$, this yields 7.13 .

Now we claim that

$$
\sum_{[i] \in \mathcal{A}}\left|G_{i}\right|=\frac{1}{2}
$$

Indeed, for every $i \in \mathbb{N}$ and $R>0$ we have

$$
\left|G_{i}\right| \geqslant\left|G_{i} \cap Q_{R}\right|=\lim _{n \rightarrow+\infty}\left|\left(E_{n}-z_{i, n}\right) \cap Q_{R}\right| .
$$

If $j$ is such that $j \sim i$ and $c_{i j} \leqslant \frac{R}{2}$, possibly enlarging $R$ we have $Q_{j, n}-z_{i, n} \subset Q_{R}$ for all $n \in \mathbb{N}$, so that

$$
\begin{aligned}
& \left|\left(E_{n}-z_{i, n}\right) \cap Q_{R}\right|=\sum_{j=1}^{I_{n}}\left|\left(E_{n}-z_{i, n}\right) \cap Q_{R} \cap\left(Q_{j, n}-z_{i, n}\right)\right| \\
& \quad \geqslant \sum_{j: c_{i j} \leqslant \frac{R}{2}}\left|\left(E_{n}-z_{i, n}\right) \cap Q_{R} \cap\left(Q_{j, n}-z_{i, n}\right)\right|=\sum_{j: c_{i j} \leqslant \frac{R}{2}}\left|\left(E_{n}-z_{i, n}\right) \cap\left(Q_{j, n}-z_{i, n}\right)\right| \\
& \quad=\sum_{j: c_{i j} \leqslant \frac{R}{2}}\left|E_{n} \cap Q_{j, n}\right|,
\end{aligned}
$$

and so

$$
\left|G_{i}\right| \geqslant \lim _{n \rightarrow+\infty}\left|\left(E_{n}-z_{i, n}\right) \cap Q_{R}\right| \geqslant \lim _{n \rightarrow+\infty} \sum_{j: c_{i j} \leqslant \frac{R}{2}}\left|E_{n} \cap Q_{j, n}\right|=\sum_{j: c_{i j} \leqslant \frac{R}{2}} \alpha_{j} .
$$

Letting $R \rightarrow+\infty$ we then have

$$
\left|G_{i}\right| \geqslant \sum_{j: i \sim j} \alpha_{j}=\sum_{j \in[i]} \alpha_{j}
$$

hence, recalling (7.11),

$$
\sum_{[i] \in \mathcal{A}}\left|G_{i}\right| \geqslant \frac{1}{2}
$$

thus proving (7.14) (since the other inequality is trivial).

We now claim that

$$
\sum_{[i] \in \mathcal{A}} P_{s}\left(G_{i}\right) \geqslant \limsup _{n \rightarrow+\infty} P_{s}\left(E_{n}\right)
$$

Indeed, by 7.14 we have that for any $\varepsilon>0$ there exist $R$, $\ell$ such that there exist $\ell$ distinct equivalence classes $\left[i_{1}\right], \ldots,\left[i_{\ell}\right] \in \mathcal{A}$ such that

$$
\frac{1}{2}-\varepsilon \leqslant \sum_{k=1}^{\ell}\left|G_{i_{k}} \cap B_{R}\right|=\lim _{n \rightarrow+\infty} \sum_{k=1}^{\ell}\left|\left(E_{n}-z_{i_{k}, n}\right) \cap B_{R}\right| .
$$


For $\rho>0$ we let

$$
E_{n, 1}^{\rho}=E_{n} \cap \bigcup_{k=1}^{\ell}\left(z_{i_{k}, n}+B_{\rho}\right) \quad E_{n, 2}^{\rho}=E_{n} \backslash E_{n, 1}^{\rho} .
$$

For $n$ sufficiently large we have that the balls $z_{i_{k}, n}+B_{R}$ are disjoint (since the $z_{i_{k}, n}$ are drifting far away from each other, being each $i_{k}$ in a different equivalence class). Therefore (7.16) gives that

$$
\left|E_{n, 1}^{R}\right| \geqslant \frac{1}{2}-2 \varepsilon \quad \text { and } \quad\left|E_{n, 2}^{R}\right| \leqslant 2 \varepsilon
$$

if $n$ is large enough. We claim that

$$
\int_{E_{n, 1}^{\bar{\rho}}} \int_{E_{n, 2}^{\bar{\rho}}} \frac{d x d y}{|x-y|^{N+s}} \leqslant \frac{c_{N}}{s(1-s)}\left|E_{n, 2}^{\bar{\rho}}\right|^{\frac{N-s}{N}} \quad \text { for some } \bar{\rho} \in\left[R, R+(2 \delta)^{-\frac{1}{N}}\right],
$$

where the constants $c_{N}, \delta$ depend only on $N$.

Indeed, if this is not the case, we would have that

$$
\begin{aligned}
& \left|E_{n, 2}^{\rho}\right|>0 \text { and } \\
& \int_{E_{n, 1}^{\rho}} \int_{E_{n, 2}^{\rho}} \frac{d x d y}{|x-y|^{N+s}}>\frac{c_{N}}{s(1-s)}\left|E_{n, 2}^{\rho}\right|^{\frac{N-s}{N}} \quad \text { for every } \rho \in\left[R, R+(2 \delta)^{-\frac{1}{N}}\right] .
\end{aligned}
$$

So we let

and we obtain

$$
\mu(\rho):=\left|E_{n, 1}^{\rho}\right|=\left|E_{n}\right|-\left|E_{n, 2}^{\rho}\right|=\frac{1}{2}-\left|E_{n, 2}^{\rho}\right|
$$

$$
\begin{aligned}
\frac{c_{N}}{s(1-s)}\left(\frac{1}{2}-\mu(\rho)\right)^{\frac{N-s}{N}} & <\int_{E_{n, 1}^{\rho}} \int_{E_{n, 2}^{\rho}} \frac{d x d y}{|x-y|^{N+s}} \\
& \leqslant \int_{E_{n, 1}^{\rho}} \int_{\mathbb{R}^{N} \backslash \bigcup_{k=1}^{\ell}\left(z_{i_{k}, n}+B_{\rho}\right)} \frac{d x d y}{|x-y|^{N+s}} \\
& \leqslant \frac{N \omega_{N}}{s} \int_{E_{n, 1}^{\rho}} \frac{d x}{\left(\rho-\left|x-z_{i_{k(x)}, n}\right|\right)^{s}} \\
& =\frac{N \omega_{N}}{s} \int_{0}^{\rho} \frac{\mu^{\prime}(z)}{(\rho-z)^{s}} d z
\end{aligned}
$$

for all $\rho \in\left[R, R+(2 \delta)^{-\frac{1}{N}}\right]$, where $k(x) \in \mathbb{N}$ is such that $x \in z_{i_{k(x)}, n}+B_{\rho}$.

From (7.20) and Lemma 4.1 (used here with $m:=1 / 2$ and $\bar{\rho}:=R$ ), we obtain that $\mu(\rho)=1 / 2$ (and so $\left.\left|E_{n, 2}^{\rho}\right|=0\right)$ for $\rho=R+(2 \delta)^{-\frac{1}{N}}$, which leads to a contradiction with 7.19 ). We thus proved (7.18). Notice that inequality (7.18) holds also with $t$ instead of $s$. So, by (7.18) and the fact that $\left|E_{n, 2}^{\rho}\right| \leqslant 2 \varepsilon$ (recall $(7.17)$ ), we obtain that

$$
\int_{E_{n, 1}^{\bar{\rho}}} \int_{E_{n, 2}^{\bar{\rho}}} \frac{d x d y}{|x-y|^{N+s}} \leqslant C \varepsilon^{\frac{N-s}{N}} \text { and } \int_{E_{n, 1}^{\bar{\rho}}} \int_{E_{n, 2}^{\bar{\rho}}} \frac{d x d y}{|x-y|^{N+t}} \leqslant C \varepsilon^{\frac{N-t}{N}},
$$

for some $C>0$, possibly depending on $n, s$ and $t$.

From this, 2.1) and (7.5) we obtain

$$
\begin{aligned}
P_{t}\left(E_{n, 1}^{\bar{\rho}}\right)+P_{t}\left(E_{n, 2}^{\bar{\rho}}\right) & =P_{t}\left(E_{n}\right)+2 \int_{E_{n, 1}^{\bar{\rho}}} \int_{E_{n, 2}^{\bar{\rho}}} \frac{d x d y}{|x-y|^{N+t}} \\
& \leqslant P_{t}\left(E_{n}\right)+C \varepsilon^{\frac{N-t}{N}} \leqslant C .
\end{aligned}
$$


Now, by (2.3), we get

$$
P_{s}\left(E_{n, 2}^{\bar{\rho}}\right) \leqslant C\left|E_{n, 2}^{\bar{\rho}}\right|^{1-\frac{s}{t}} P_{t}\left(E_{n, 2}^{\bar{\rho}}\right)^{\frac{s}{t}},
$$

up to renaming $C$. Using this, 7.22 and then 7.17 once more, and possibly renaming $C$ again, we conclude that

$$
\begin{aligned}
P_{s}\left(E_{n, 2}^{\bar{\rho}}\right) & \leqslant C\left|E_{n, 2}^{\bar{\rho}}\right|^{1-\frac{s}{t}}\left(P_{t}\left(E_{n, 1}^{\bar{\rho}}\right)+P_{t}\left(E_{n, 2}^{\bar{\rho}}\right)\right)^{\frac{s}{t}} \\
& \leqslant C\left|E_{n, 2}^{\bar{\rho}}\right|^{1-\frac{s}{t}} \\
& \leqslant C \varepsilon^{1-\frac{s}{t}} .
\end{aligned}
$$

Consequently, using (2.1) and (7.21), we conclude that

$$
\begin{aligned}
P_{s}\left(E_{n, 1}^{\bar{\rho}}\right) & =P_{s}\left(E_{n}\right)-P_{s}\left(E_{n, 2}^{\bar{\rho}}\right)+2 \int_{E_{n, 1}^{\bar{\rho}}} \int_{E_{n, 2}^{\bar{\rho}}} \frac{d x d y}{|x-y|^{N+s}} \\
& \geqslant P_{s}\left(E_{n}\right)-C \varepsilon^{1-\frac{s}{t}} .
\end{aligned}
$$

Also, from (7.5), (7.12) and the compact embedding of $H^{\frac{t}{2}}$ into $H^{\frac{s}{2}}$ (see [16, Section 7]), we see that

$$
\lim _{n \rightarrow+\infty} P_{s}\left(\left(E_{n}-z_{i_{k}, n}\right) \cap B_{\bar{\rho}}\right)=P_{s}\left(G_{i_{k}} \cap B_{\bar{\rho}}\right) .
$$

Now we recall that if $K$ is a convex set, then $P_{s}(E \cap K) \leqslant P_{s}(E)$ (see for instance [18, Lemma B.1]). Together with 2.2 and 7.23 , this implies

$$
\begin{aligned}
\sum_{[i] \in \mathcal{A}} P_{s}\left(G_{i}\right) & \geqslant \sum_{k=1}^{\ell} P_{s}\left(G_{i_{k}} \cap B_{\bar{\rho}}\right) \\
& =\lim _{n \rightarrow+\infty} \sum_{k=1}^{\ell} P_{s}\left(\left(E_{n}-z_{i_{k}, n}\right) \cap B_{\bar{\rho}}\right) \\
& \geqslant \lim _{n \rightarrow+\infty} P_{s}\left(E_{n, 1}^{\bar{\rho}}\right) \\
& \geqslant \limsup _{n \rightarrow+\infty} P_{s}\left(E_{n}\right)-C(N, s, t) \varepsilon^{\frac{t-s}{t}},
\end{aligned}
$$

which gives 7.15 by letting $\varepsilon \rightarrow 0^{+}$.

From 7.13 and 7.15 we obtain that

$$
\frac{\sum_{[i] \in \mathcal{A}}(1-t) P_{t}\left(G_{i}\right)}{\left(s \sum_{[i] \in \mathcal{A}} P_{s}\left(G_{i}\right)\right)^{\frac{N-t}{N-s}}} \leqslant \liminf _{n \rightarrow+\infty} \frac{(1-t) P_{t}\left(E_{n}\right)}{\left(s P_{s}\left(E_{n}\right)\right)^{\frac{N-t}{N-s}}} .
$$

Let us now prove that the there exists $j$ such that

$$
\frac{(1-t) P_{t}\left(G_{j}\right)}{\left(s P_{s}\left(G_{j}\right)\right)^{\frac{N-t}{N-s}}} \leqslant \frac{\sum_{[i] \in \mathcal{A}}(1-t) P_{t}\left(G_{i}\right)}{\left(s \sum_{[i] \in \mathcal{A}} P_{s}\left(G_{i}\right)\right)^{\frac{N-t}{N-s}}}=: S .
$$


Indeed, if it is not the case, we get

$$
\begin{aligned}
S & =\frac{\sum_{[i] \in \mathcal{A}}(1-t) P_{t}\left(G_{i}\right)}{\left(s \sum_{[i] \in \mathcal{A}} P_{s}\left(G_{i}\right)\right)^{\frac{N-t}{N-s}}}=\frac{\sum_{[i] \in \mathcal{A}}\left(\frac{(1-t) P_{t}\left(G_{i}\right)}{\left(s P_{s}\left(G_{i}\right)\right)^{\frac{N-t}{N-s}}}\right)\left(s P_{s}\left(G_{i}\right)\right)^{\frac{N-t}{N-s}}}{\left(s \sum_{[i] \in \mathcal{A}} P_{s}\left(G_{i}\right)\right)^{\frac{N-t}{N-s}}} \\
> & S \frac{\sum_{[i] \in \mathcal{A}}\left(s P_{s}\left(G_{i}\right)\right)^{\frac{N-t}{N-s}}}{\left(s \sum_{[i] \in \mathcal{A}} P_{s}\left(G_{i}\right)\right)^{\frac{N-t}{N-s}}} \geqslant S,
\end{aligned}
$$

which is impossible. To get the last estimate we used the elementary inequality $\left(\sum_{i} c_{i}\right)^{\alpha} \leqslant \sum_{i} c_{i}^{\alpha}$ which holds true for $c_{i} \geqslant 0$ and $\alpha \in(0,1)$.

Now, let $j$ be the index satisfying (7.26). Then, by 7.25 we get

$$
\frac{(1-t) P_{t}\left(G_{j}\right)}{\left(s P_{s}\left(G_{j}\right)\right)^{\frac{N-t}{N-s}}} \leqslant \liminf _{n \rightarrow \infty} \frac{(1-t) P_{t}\left(E_{n}\right)}{\left(s P_{s}\left(E_{n}\right)\right)^{\frac{N-t}{N-s}}} .
$$

Then, given any set $E$, fixed any $\epsilon>0$, we intersecate $E$ with a big ball $B_{R_{\epsilon}}$ in such a way that

$$
\frac{(1-t) P_{t}\left(E \cap B_{R_{\epsilon}}\right)}{\left(s P_{s}\left(E \cap B_{R_{\epsilon}}\right)\right)^{\frac{N-t}{N-s}}} \leqslant \frac{(1-t) P_{t}(E)}{\left(s P_{s}(E)\right)^{\frac{N-t}{N-s}}}+\epsilon .
$$

Then, by the minimality of $E_{n}$,

$$
\frac{(1-t) P_{t}\left(E \cap B_{R_{\epsilon}}\right)}{\left(s P_{s}\left(E \cap B_{R_{\epsilon}}\right)\right)^{\frac{N-t}{N-s}}} \geqslant \frac{(1-t) P_{t}\left(E_{n}\right)}{\left(s P_{s}\left(E_{n}\right)\right)^{\frac{N-t}{N-s}}}
$$

for any $n \geqslant n_{\epsilon}$. Thus, by (7.27),

$$
\frac{(1-t) P_{t}(E)}{\left(s P_{s}(E)\right)^{\frac{N-t}{N-s}}}+\epsilon \geqslant \liminf _{n \rightarrow \infty} \frac{(1-t) P_{t}\left(E_{n}\right)}{\left(s P_{s}\left(E_{n}\right)\right)^{\frac{N-t}{N-s}}} \geqslant \frac{(1-t) P_{t}\left(G_{j}\right)}{\left(s P_{s}\left(G_{j}\right)\right)^{\frac{N-t}{N-s}}} .
$$

By sending $\epsilon \searrow 0$ we see that $G_{j}$ is the desired minimizer, which concludes the proof.

Proposition 7.6. Let $F$ be a minimizer of (7.1). Then $F$ is a multiplicative $\omega$-minimizer of the $t$-perimeter, that is, for any set $E$ such that $F \Delta E \subset B(x, R)$, there holds

$$
P_{t}(F, B(x, R)) \leqslant\left(1+C R^{t-s}\right) P_{t}(E, B(x, R)) \quad \text { for any } R<R_{0},
$$

where $R_{0}, C$ depend only on $N, \delta_{0}$ and $|F|$.

Proof. First, if $\alpha \in(0,1)$, by graphic the functions, one sees that, for any $r \geqslant 0$,

$$
1-r^{\alpha} \leqslant|1-r| .
$$

Also, from (5.11), we know that

$$
P_{s}(F)-P_{s}(E) \leqslant P_{s}(F \Delta E),
$$

for any sets $E$ and $F$, and so, by possibly exchanging the roles of $E$ and $F$ we obtain

$$
\left|P_{s}(E)-P_{s}(F)\right| \leqslant P_{s}(F \Delta E)
$$


Now, letting $E$ be such that $F \Delta E \subset B(x, R)$, using the minimality of $F,(7.28)$ and 7.29 we see that

$$
\begin{aligned}
P_{t}(E) & \geqslant P_{s}(E)^{\frac{N-t}{N-s}} \frac{P_{t}(F)}{P_{s}(F)^{\frac{N-t}{N-s}}} \\
& =P_{t}(F)+\left(\frac{P_{s}(E)^{\frac{N-t}{N-s}}}{P_{s}(F)^{\frac{N-t}{N-s}}}-1\right) P_{t}(F) \\
& \geqslant P_{t}(F)-\left|\frac{P_{s}(E)}{P_{s}(F)}-1\right| P_{t}(F) \\
& \geqslant P_{t}(F)-\frac{P_{t}(F)}{P_{s}(F)}\left|P_{s}(E)-P_{s}(F)\right| \\
& \geqslant P_{t}(F)-\frac{P_{t}(F)}{P_{s}(F)} P_{s}(F \Delta E) .
\end{aligned}
$$

Hence, by applying the fractional isoperimetric inequality (2.15) to $P_{s}(F)$, we obtain that

$$
P_{t}(E) \geqslant P_{t}(F)-C\left(N, \delta_{0}\right)|F|^{-\frac{N-s}{N}} P_{s}(F \Delta E) .
$$

As in (5.15), by means of (2.3) and again the fractional isoperimetric inequality we then get

$$
\begin{aligned}
P_{t}(E, B(x, R)) & \geqslant P_{t}(F, B(x, R))-C\left(N, \delta_{0}\right)|F|^{-\frac{N-s}{N}}|F \Delta E|^{\frac{t-s}{N}} P_{t}(F \Delta E) \\
& =\left(1-C\left(N, \delta_{0}\right)|F|^{-\frac{N-s}{N}} R^{t-s}\right) P_{t}(F, B(x, R)),
\end{aligned}
$$

which gives

$$
P_{t}(F, B(x, R)) \leqslant \frac{|F|^{-\frac{N-s}{N}}}{1-C\left(N, \delta_{0}\right) R^{t-s}} P_{t}(E, B(x, R)) .
$$

Reasoning as in Section 5, from Proposition (7.6) we obtain the following regularity result.

Corollary 7.7. There exists $\beta=\beta\left(N, \delta_{0}\right)<1$ such that any minimizer $F$ of $(7.1)$ is bounded and has boundary of class $C^{1, \beta}$, outside of a closed singular set of Hausdorff dimension at most $N-2$ (respectively $N-8$ if $t=1)$.

Proof of Theorem 1.4. The existence claim is a consequence of Theorem 7.2 and the regularity follows from Corollary 7.7 .

\section{REFERENCES}

[1] E. Acerbi, N. Fusco, M. Morini, Minimality via second variation for a nonlocal isoperimetric problem. Comm. Math. Phys., 322 (2013), 515-557.

[2] L. Ambrosio, V. Caselles, S. Masnou, J.-M. Morel, Connected components of sets of finite perimeter and applications to image processing. J. Eur. Math. Soc. (JEMS), 3 (2001), no. 1, 39-92.

[3] L. Ambrosio, G. De Philippis, L. Martinazzi, $\Gamma$-convergence of nonlocal perimeter functionals. Manuscripta Math., 134 (2011), 377-403.

[4] E. Bombieri, Regularity theory for almost minimal currents. Arch. Rational Mech. Anal., 78 (1982), 99130.

[5] M. Bonacini, R. Cristoferi, Local and global minimality results for a nonlocal isoperimetric problem on $\mathbb{R}^{N}$. To appear in SIAM J. Math. Anal..

[6] J. Bourgain, H. Brezis, P. Mironescu, Another look at Sobolev spaces, Optimal Control and Partial Differential Equations, J. L. Menaldi, E. Rofman, A. Sulem (Eds.), a volume in honor of A. Bensoussan's 60th birthday, IOS Press, Amsterdam, 2001, 439-455. 
[7] J. Bourgain, H. Brezis, P. Mironescu, Limiting embedding theorems for $W^{s, p}$ when $s \nearrow 1$ and applications. J. Anal. Math., 87 (2002), 77-101.

[8] L. Brasco, E. Lindgren, E. Parini, The fractional Cheeger problem. Preprint (2013). Available at http://cvgmt.sns.it/paper/2225/

[9] L. Caffarelli, J. M. Roquejoffre, O. Savin, Non-local minimal surfaces. Comm. Pure Appl. Math., 63 (2010), 1111-1144.

[10] L. Caffarelli, E. Valdinoci, Uniform estimates and limiting arguments for nonlocal minimal surfaces. Calc. Var. Partial Differential Equations, 41 (2011), no. 1-2, 203-340.

[11] L. Caffarelli, E. Valdinoci, Regularity properties of nonlocal minimal surfaces via limiting arguments. Adv. Math., 248 (2013), 843-871.

[12] M. C. Caputo, N. Guillen, Regularity for non-local almost minimal boundaries and applications. Preprint (2011). Available at http://arxiv.org/pdf/1003.2470.

[13] M. Cicalese, E. Spadaro, Droplet minimizers of an isoperimetric problem with longe-range interactions. Comm. Pure Appl. Math., 66 (2013), 1298-1333.

[14] J. DÁvila, On an open question about functions of bounded variation. Calc. Var. Partial Differential Equations, 15 (2002), no. 4, 519-527.

[15] J. DÁvila, M. Del Pino, J. Wei, Nonlocal s-minimal surfaces and Lawson cones. Preprint (2014). Available at http://arxiv.org/abs/1402.4173

[16] E. Di Nezza, G. Palatucci, E. Valdinoci, Hitchhiker's quide to the frctional Sobolev Spaces. Bull. Sci. Math., 136 (2012), 521-573.

[17] S. Dipierro, A. Figalli, G. Palatucci, E. Valdinoci, Asymptotics of the s-perimeter as $s \searrow 0$, Discrete Contin. Dyn. Syst., 33 (2013), no. 7, 2777-2790.

[18] A. Figalli, N. Fusco, F. Maggi, V. Millot, M. Morini, Isoperimetry and stability properties of balls with respect to nonlocal energies. Preprint (2014). Available at http://cvgmt.sns.it/paper/2380/,

[19] R.L. Frank, E.H. Lieb, R. Seiringer, Hardy-Lieb-Thiring inequalities for fractional schrödinger operators. J. Amer. Math. Soc., 21 (2008), 925-950.

[20] R.L. Frank, R. Seiringer, Non-linear ground state representations and sharp Hardy inequalities. J. Funct. Anal., 255 (2008), 3407-3430.

[21] B. Fuglede, Stability in the isoperimetric problem for convex or nearly spherical domains in $\mathbb{R}^{n}$. Trans. Amer. Math. Soc., 314 (1989), 619-638.

[22] N. Fusco, V. Julin, On the regularity of critical and minimal sets of a free interface problem, arXiv preprint arXiv:1309.6810 (2013).

[23] N. Fusco, F. Maggi, A. Pratelli, The sharp quantitative isoperimetric inequality. Ann. of Math., 168 (2008), 941-980.

[24] N. Fusco, V. Millot, M. Morini, A quantitative isoperimetric inequality for fractional perimeters. J. Funct. Anal., 26 (2011), 697-715.

[25] M. Goldman, M. Novaga, Volume-constrained minimizers for the prescribed curvature problem in periodic media. Calc. Var. Partial Differential Equations, 44 (2012), no. 3, 297-318.

[26] M. Goldman, M. Novaga, B. Ruffini, Existence and stability for a non-local isoperimetric model of charged liquid drops, Preprint (2013).

[27] V. Julin, Isoperimetric problem with a Coulombic repulsive term. To appear in Indiana Univ. Math. J..

[28] H. Knüpfer, C. B. Muratov, On an Isoperimetric Problem with a Competing Nonlocal Term I: The planar case. Comm. Pure Appl. Math., 66 (2013), 1129-1162.

[29] H. Knüpfer, C. B. Muratov, On an Isoperimetric Problem with a Competing Nonlocal Term II: The general case. Comm. Pure Appl. Math., doi:10.1002/cpa.21479.

[30] F. MAGgi, Sets of finite perimeter and geometric variational problems. An introduction to Geometric Measure Theory. Cambridge Studies in Advanced Mathematics 135, Cambridge University Press, 2012.

[31] V. Maz'ya, T. Shaposhnikova, On the Bourgain, Brezis, and Mironescu theorem concerning limiting embeddings of fractional Sobolev spaces. J. Funct. Anal., 195 (2002), no. 2, 230-238.

[32] S. G. SAmко, Hypersingular integrals and their applications. Analytical Methods and Special Functions. Taylor \& Francis, Ltd., London, 2002.

[33] O. Savin, E. Valdinoci, Г-convergence for nonlocal phase transitions. Ann. Inst. H. Poincaré Anal. Non Linèaire, 29 (2012), 479-500. 
[34] O. Savin, E. Valdinoci, Regularity of nonlocal minimal cones in dimension 2. Calc. Var. Partial Differential Equations, 48 (2013), no. 1-2, 33-39.

[35] I. Tamanini, Boundaries of Caccioppoli sets with Hölder-continuous normal vector. J. Reine Angew. Math., 334 (1982), 27-39.

[36] A. Visintin, Generalized coarea formula and fractal sets. Japan J. Indust. Appl. Math., 8 (1991), no. 2, 175-201.

E-mail address, Agnese Di Castro: dicastro@mail.dm.unipi.it

E-mail address, Matteo Novaga: novaga@dm.unipi.it

E-mail address, Berardo Ruffini: berardo.ruffini@ujf-grenoble.fr

E-mail address, Enrico Valdinoci: enrico.valdinoci@wias-berlin.de

(A. Di Castro, M. Novaga) Dipartimento di Matematica, Università di Pisa Largo Bruno Pontecorvo, 5, 56127 Pisa, Italy

(B. Ruffini) Université Joseph Fourier, BP 53, 38041, Grenoble Cedex 39, France

(E. Valdinoci) Weierstrass Institute, Mohrenstrasse 39, 10117 Berlin, Germany 